\title{
Numerical modeling of existing acoustic emission sensor absolute calibration approaches
}

\author{
MARKUS G. R. SAUSE ${ }^{1}$, MARVIN A. HAMSTAD \\ ${ }^{1}$ University of Augsburg, Institute for Materials Resource Management, Mechanical Engineering, D-86135 \\ Augsburg, Germany \\ 2University of Denver, Daniel Felix Ritchie School of Engineering and Computer Science, Denver, CO, USA \\ 80208
}

\begin{abstract}
Within recent years, several alternative approaches to acoustic emission sensor calibration have been proposed. As some of these approaches make use of new geometries and materials, the aspect of absolute calibration in the context of these proposals is evaluated in this contribution. Validated numerical methods are applied to compare the expected acoustic emission sensor sensitivity in a variety of material and geometry configurations. The numerical approach uses a coupled structural and piezoelectric formulation in combination with electric circuit modeling. Established routines allow obtaining the sensitivity versus frequency curve as sensor response in electrical voltage per meters displacement. This study is performed for one previously used conical sensor design and one typical disc type sensor design to examine the impact of the investigated approaches for these different sensor dimensions and designs. Specific attention is paid to the influence of the propagation medium material properties, the chosen wave type and the geometry of the propagation medium. For use of plate-like propagation media further aspects, such as thickness, distance and formation of guided wave modes are evaluated. A primary result is that there is a significant influence to be expected for calibration attempts with different wave types for the same material, which can be of particular relevance when using sensor systems with extended aperture, such as common to most of the commercial sensors.
\end{abstract}

\section{Introduction}

Early complete methods for the absolute calibration of acoustic emission (AE) sensors came quite a few years after the first commercial AE sensors were available in the late 1960s. The first publication in 1976 described a reciprocity-based method developed in Japan [1]. The approach used electrical signals to generate waves. The second publication in 1982 described an alternate method developed in the United States based on waves generated by the fracture of a glass capillary [2]. Both of these methods relied on the use of very large steel blocks as the wave transmission medium. The large blocks were used to eliminate reflections from the boundaries. In the case of both methods, the waves present were limited to bulk waves and Rayleigh waves. The first method was adopted as a standard NDIS 2109 in 1991, and the second method was adopted as a standard ASTM E1106 in 1986. Later approaches in the United Kingdom started to evaluate the use of laser vibrometers for signal referencing [3] instead of the capacitive sensor used before [4]. Recently two new geometries have been proposed. These both use guided waves. In one case, plate geometry is used [5] as the wave propagation media and in the other case, rod geometry is used [6] as the wave propagation media. Apart from these approaches, the direct coupling of the sensor under test and an actuator, commonly referred to as face-to-face method, is frequently used. The shortcomings of this method are extensively discussed in [7], so these are not repeated here. The above brief history of sensor calibration (with associated experiments) is not intended as a comprehensive review that would include the many follow-on works. 
This work is directed not towards calibration with associated experiments but at modeling wave-propagation in media along with modeling the associated sensor calibrations. This modeling approach has several benefits, but also some drawbacks. On the beneficial side, the modeling approach provides very good control over experimentally inaccessible parameters and allows evaluation of all involved physical quantities at arbitrary locations. Also, the visualization of the wave propagation by itself is helpful to understand and discuss boundary and geometry effects on the recorded AE signals. In addition, an initial modeling of calibration may avoid costs to carry out extensive experimental campaigns. However, the main potential drawback certainly is the reliability of the modeling procedure. Throughout several previous publications we have established a numerical routine, which is capable of predicting the $A E$ sensor response in sensor calibration situations [8]-[10]. The overall feasibility of such approaches has also been shown by other groups (e.g. in [11], [12]) for direct epicenter wave front arrivals after short distance of propagation $(<12.7 \mathrm{~mm})$ with a signal duration allowing multiple reflections from the bottom to top of the plate propagation medium. In this study, the modeling approach uses a propagation distance of $100 \mathrm{~mm}$ for the propagation media. With reference to established standards, the size of the propagation media is chosen to allow detection free of reflections. Then four cases are considered for two very different sensors. Subsequently, the principle findings of this study are presented and several factors of influence, such as wave propagation mode(s), choice of material, sensor design, and implications of using Lamb waves are elucidated. Particular emphasis is given to elaborate the use of Lamb waves for potential use for $A E$ absolute sensor calibration procedures. If such a propagation medium could be used, it would make it much easier to obtain and use such a medium. No focus is given to the face-to-face approach, as we do not consider this an absolute calibration method.

\section{Modeling approach}

All numerical computations are carried out within the software program Comsol Multiphysics 5.2a using the "Structural Mechanics Module" and the "AC/DC Module". The software program uses an implicit formulation in order to solve transient coupled partial differential equations. The principle settings follow the validated approach established in [9] and apply the full 3D coupled piezoelectric equations in combination with a P-SPICE circuit simulation to account for the attached electronics such as preamplifiers and acquisition electronics.

\subsection{Model configuration}

Within the numerical framework, four principle case studies for AE sensor calibration are investigated. These are summarized in Fig. 1, which includes their dimensions. All material properties used are listed in Tab. 1.

\section{Model geometries}

The first case considers the typical arrangement with a test source opposite to the test sensor, separated by a large cylinder-type propagation medium. As test source function, an emulation of the rise time of a glass capillary fracture is used. This is done by a force function applied at a circular contact area with radius $r_{s}=0.5 \mathrm{~mm}$. The force is applied in the z-direction using a cosine-bell shape function reaching a maximum $F_{m}=10 \mathrm{~N}$ within a rise-time $t_{s}=0.3 \mu \mathrm{s}$ [13], [14] following the values in the current version of ASTM E1106-12: 
$F(t)=\left\{\begin{array}{cc}\frac{F_{m}}{2} \cdot\left(1-\cos \left(\frac{\pi t}{t_{s}}\right)\right) & t \leq t_{s} \\ 1 & t>t_{s}\end{array}\right.$

The $A E$ sensor under investigation is located at $100 \mathrm{~mm}$ opposite (epicenter) to the source position with the $A E$ sensor geometries found in Fig. 2. In this configuration, the wave propagation mode is solely longitudinal and transversal waves arriving at the sensor position. The radius of the cylinder is large enough to avoid interference of reflected waves at the $A E$ sensor position within the first $50 \mu \mathrm{s}$ for a steel block. As the geometry of the first case is fully axisymmetric, the computation was carried out with a central axis of symmetry.

For the second case, the radius of the propagation medium is reduced to yield a typical rod configuration. While all other settings are kept identical to the first case, the change in boundary constraints now forces a guided wave propagation mode in the form of rod waves. Again, the computation was carried out in an axisymmetric geometry. Due to the symmetry axis at $\mathrm{x}=0 \mathrm{~mm}$, the only allowed guided wave modes for frequencies up to $1 \mathrm{MHz}$ are the $L(0,1), L(0,2), L(0,3)$ and $L(0,4)$ rod wave modes for the source position at $x=0 \mathrm{~mm}$. The 100 $\mathrm{mm}$ length of the rod was such that no end reflections were present in the first $55 \mu$ s for a steel rod.

As a third case, the arrangement of test source and AE sensor is changed relative to the first case. As seen in Fig. 1-c, the test source function is now applied at the top of the cylinder with the center of the AE sensor offset by $100 \mathrm{~mm}$. In this configuration, the test source function of equation (1) is applied in the minus z-direction. Besides the longitudinal and transversal waves, this source also initiates a Rayleigh wave spreading on the top surface of the cylinder. In this configuration, the Rayleigh wave is the dominant wave mode detected by the $A E$ sensor. Due to the break of symmetry, it is no longer feasible to model the configuration by an axisymmetric approach. Instead, the "general extrusion coupling feature" of Comsol Multiphyiscs was applied to link a 2D wave propagation model with a $3 D$ half-volume $A E$ sensor representation (see below for details of 2D-3D coupling). The dimensions of the cylinder were such that no significant reflections were present in the first $68 \mu$ s for a steel cylinder.

The fourth case is a direct extension of the third case. Keeping all settings identical, the thickness of the cylinder was reduced to yield a thin plate of $3 \mathrm{~mm}$ thickness. Accordingly, the wave propagation from the test source to the sensor changes, and the wave travels as distinct Lamb wave modes due to the large ratio of the propagation distance of $100 \mathrm{~mm}$ to the plate thickness. For the frequency range up to $1 \mathrm{MHz}$, these modes are the antisymmetric $\left(A_{0}\right.$ and $A_{1}$ ) and symmetric $\left(S_{0}\right.$ and $S_{1}$ ) Lamb wave modes. For a steel plate, the outer radius was such that no edge reflections were present during the first $70 \mu \mathrm{s}$. 
(a)
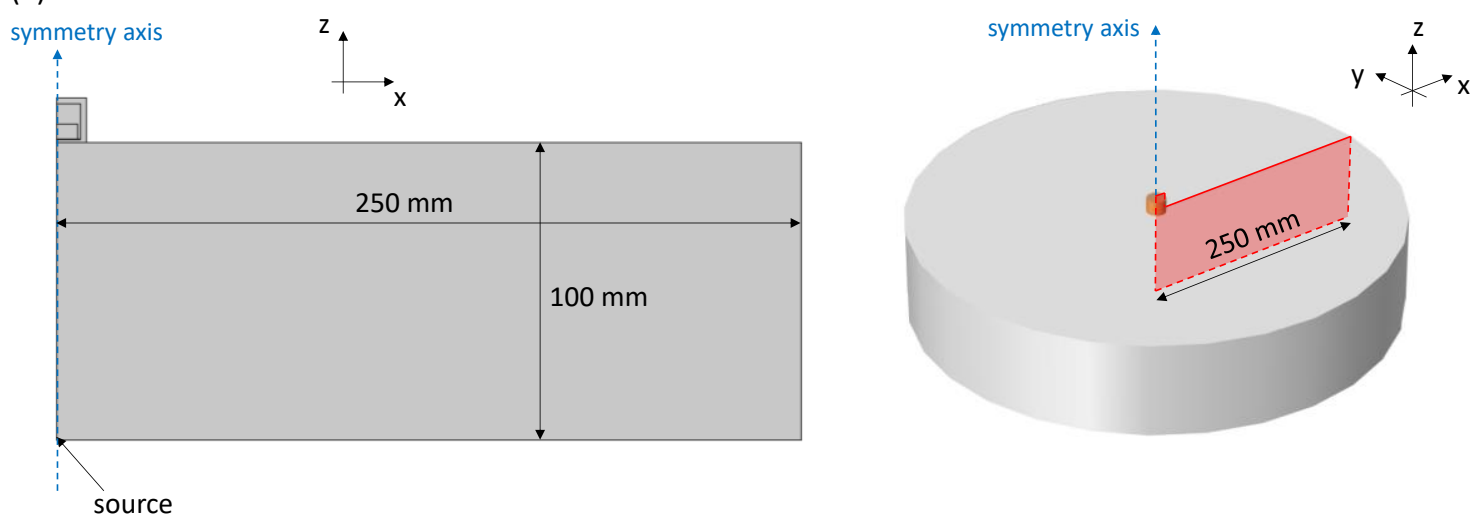

(b)

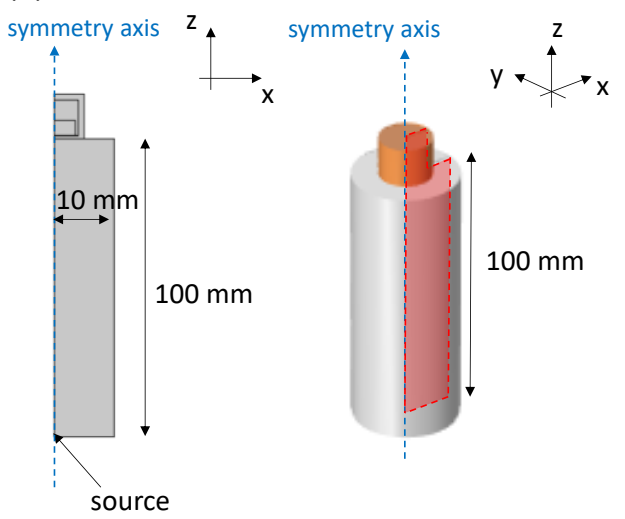

(c)
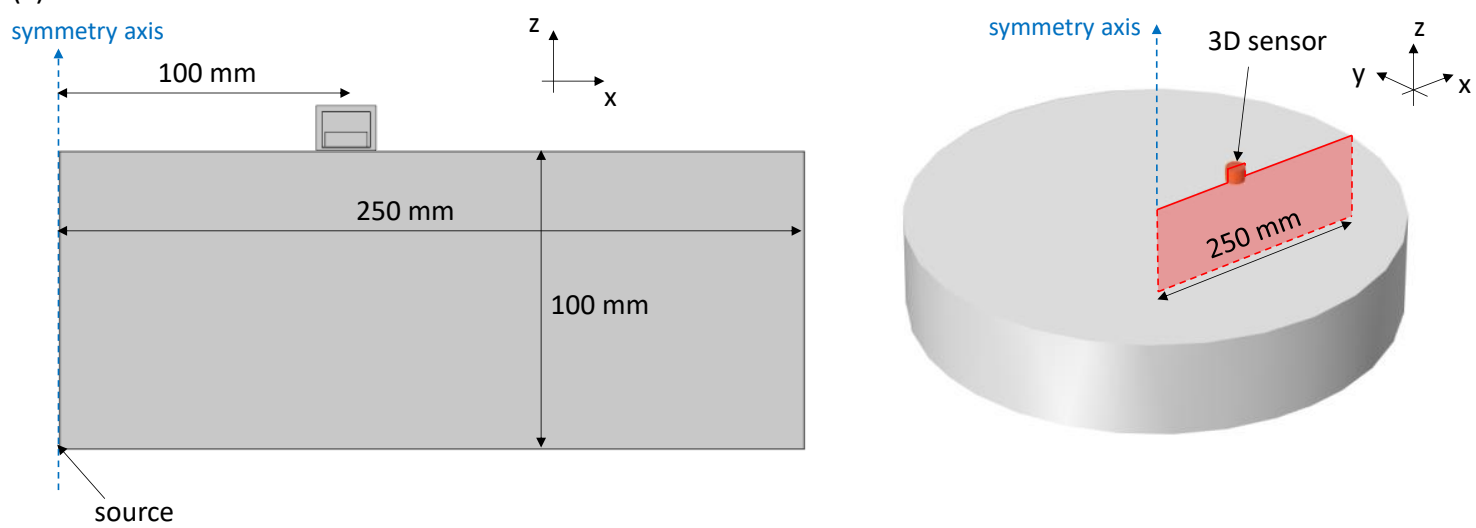

(d)
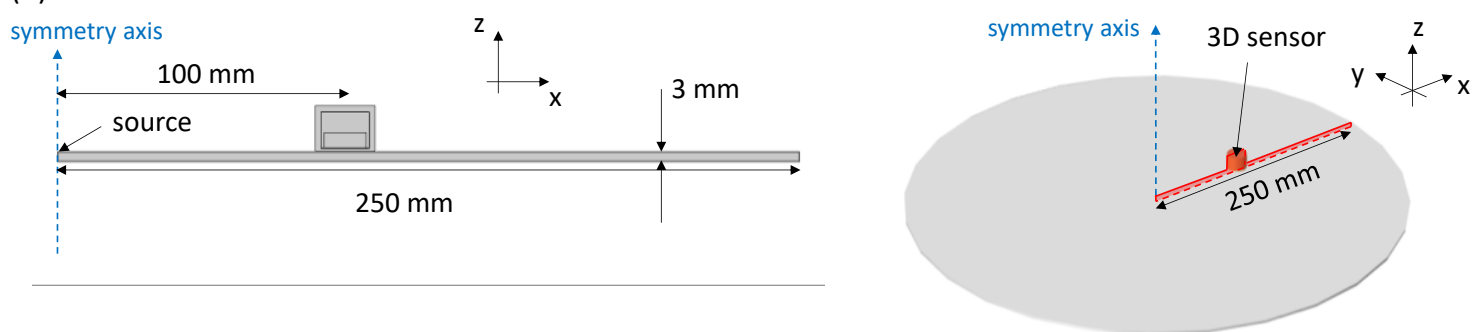

Fig. 1. Basic geometries of different configurations (prior to variations) evaluated as used for longitudinal and transversal waves (a), rod waves (b), Rayleigh waves (c) and Lamb waves (d). Modeled cross-sections and volumes are marked in red; generic AE sensor is used as example.

\section{Sensor implementation}

For this study, two representative AE sensor models were chosen. As first choice, a cone shaped PZT-5A element with brass backing mass, representative of typical conical sensors was 
selected. The dimensions and implementation of this AE sensor model is consistent with the settings reported in [9]. As second choice, a cylindrical, disc-type PZT-5A element with epoxy adhesive bonding, ceramic wear plate and metallic sensor casing was used. The dimensions of these elements conform to typical values found in commercial resonant type AE sensors. In both cases, the electrical ground condition is chosen at the base of the piezoelectric element and signal connection to the P-SPICE circuit simulation was made by terminal boundary conditions evaluating the top surface of the piezoelectric element as an electrical equipotential plane. All other surfaces of the PZT element are set as zero charge Neumann boundary conditions. Besides symmetry conditions at the xz-cross-section plane, no mechanical boundary constraints were set. To account for the influence of the electric impedance of the experimentally attached signal cables and preamplifiers, the P-SPICE circuit model was chosen identical to [9], [10] to mimic a typical experimental condition. The computed voltage across the load resistor of the modeled preamplifier is used for AE signal evaluation. No additional preamplifier gain is included in the reported values, i.e. the preamplifier gain was chosen as one.

(a)

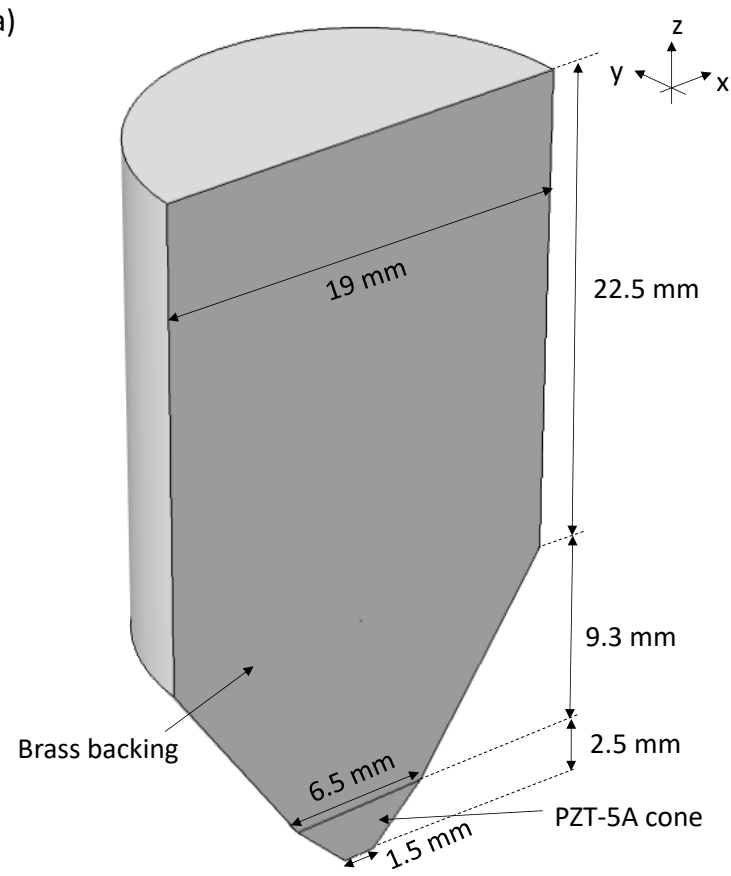

(b)

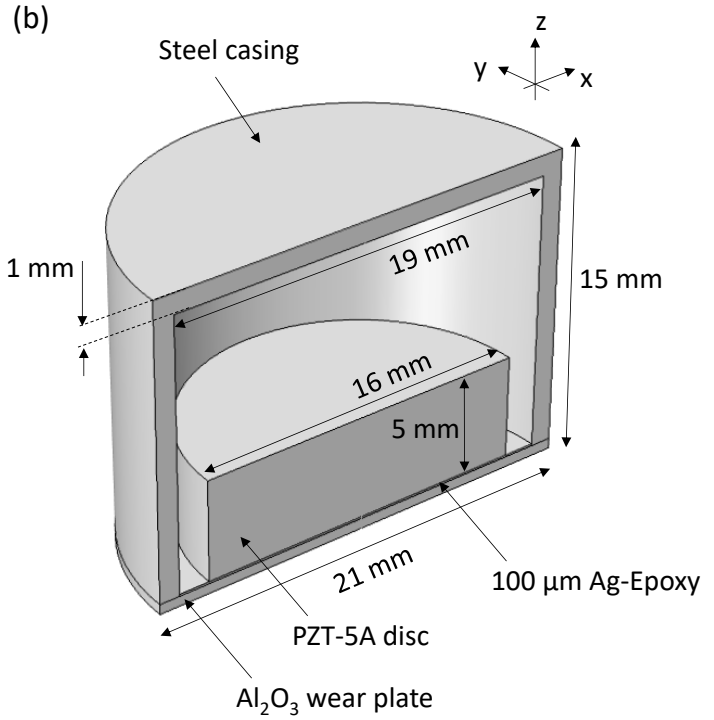

Fig. 2. Geometry of conical type AE sensor (a) following validated approach of [9] and geometry of disc type AE sensor (b).

\section{D-3D coupling}

To reduce the computational intensity of the models, an approach was chosen to model the wave propagation in the test medium using $2 \mathrm{D}$ codes with the $\mathrm{AE}$ sensor model coupled as a 3D model. This bi-directional coupling using the "general extrusion operator" feature of Comsol Multiphysics maps the contact line values of the 2D model to the contact surface of the 3D sensor model taking into account the radial position at the contact surface and the boundary constraints from both sides (sensor face and propagation medium). Given the small maximum opening angle $\alpha=6.0^{\circ}$ as defined in Fig. 3-b, no mapping correction was made for the angular deviation from the source position to the sensor. However, this mapping requires correcting the computed signal voltages, for the change in geometric dimension from $2 \mathrm{D}$ to $3 \mathrm{D}$, to provide results consistent with the purely 2D cases. The 3D sensor as implemented uses one xz-symmetry plane, so the contact face is a half-circle of area 
$\mathrm{A}_{\mathrm{C}}=\frac{1}{2} \cdot\left(\frac{d}{2}\right)^{2} \cdot \pi=\frac{1}{2} \cdot d^{2} \cdot \frac{\pi}{4}$

Because of the orthogonal direction of the extrusion coupling operator, the virtual extrusion surface is a rectangle with area

$A_{R}=\frac{1}{2} \cdot d^{2}$

Thus, the mismatch in both areas evaluates as a factor $\frac{\pi}{4}$. Because of the chosen symmetry plane, this factor needs to be multiplied by a factor of two, in order to return the result of a full volume 3D sensor. Thus to compare with the purely axisymmetric cases these coupled results where multiplied by a factor $\pi / 2$. The equality of computed $A E$ sensor signals in a full $3 \mathrm{D}$ approach and in the described 2D-3D coupled approach is shown in Fig. 3-c for the Lamb wave case. For the 2D axisymmetric code, the contact condition at the interface between sensor and propagation medium uses the default "continuity" assumption in Comsol Multiphysics. This is a rigid coupling of mesh nodes of both geometries, allowing displacement continuity along this interface. Similar to the 2D axisymmetric code, the coupling at the interface between sensor face and propagation medium for the 2D-3D coupled approach is done as a continuity assumption (physically this would be a very thin bond between the propagation media and the sensor face), i.e. the full 3D ( $x, y, z)$-displacement vector is mapped bi-directionally.

(a)

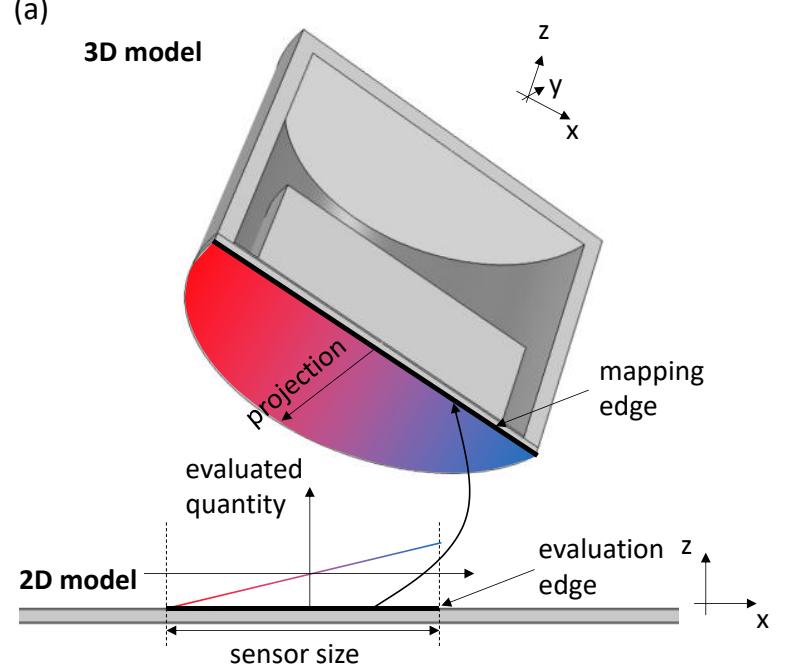

(b)

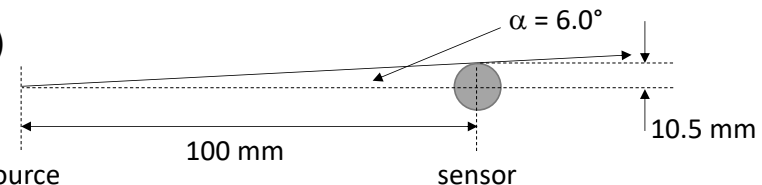

(c)

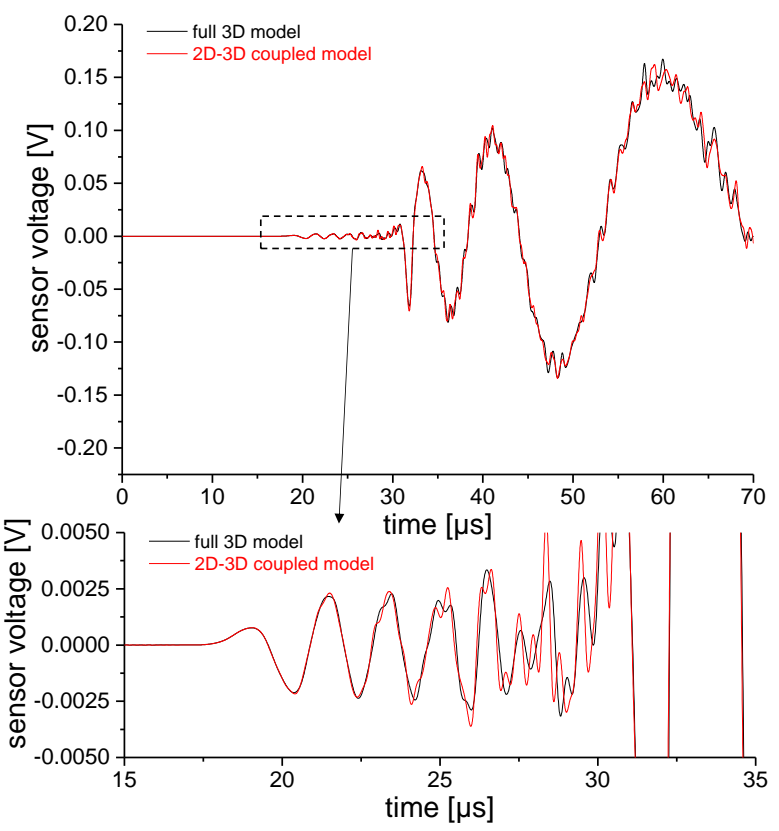

Fig. 3. Schematic of the 2D-3D coupling (a), discussion of opening angle (b) and comparison of $A E$ signals of full 3D approach and 2D-3D coupled approach corrected by $\pi / 2$ (c).

\section{Discretization settings}

All numerical settings are based on the detailed evaluation of numerical parameters in previous studies [9], [10], [15]. Therein, the suitability of these settings was established by means of comparison to analytical curves, experimental data and other numerical code results. For the present study a maximum mesh edge length of $0.25 \mathrm{~mm}$ for a Delaunay tessellation [16] to generate the meshes proved sufficient to investigate the bandwidth up to 
1.5 $\mathrm{MHz}$ following the rule of mesh size to be $\leq \lambda / 4$ with $\lambda$ being the shortest relevant wavelength. For the transient computation, the generalized- $\alpha$ solver scheme [17] with $5 \mathrm{~ns}$ time step was applied. For each model, a total duration of $75 \mu$ s (steel and aluminum) or 150 us (PMMA) after signal excitation was computed for the original cases.

\section{Material properties}

All domains use the material properties found in Tab. 1 and Tab. 2 as indicated in Figs. 1 and 2. For the PZT element, z-direction was chosen as polarization direction. In order to take into account the viscoelastic nature of PMMA, a Rayleigh damping approach was applied with mass damping factor $\alpha=1 \cdot 10^{3}$ and stiffness damping factor $\beta=1 \cdot 10^{-10}$. This material model results in attenuation values of about $2 \mathrm{~dB} / \mathrm{cm}$ at $2 \mathrm{MHz}$ frequency, which is in good agreement with experimental values reported by [18].

\begin{tabular}{|l|l|l|l|}
\hline Name & Density $\left[\mathrm{kg} / \mathbf{m}^{\mathbf{3}}\right]$ & Modulus [GPa] & Poisson's ratio \\
\hline Steel & 7800 & 210.9 & 0.33 \\
\hline $\begin{array}{l}\text { Polymethylmethacrylate } \\
\text { (PMMA) }\end{array}$ & 1160 & 6.2 & 0.32 \\
\hline Aluminum & 2660 & 70.0 & 0.33 \\
\hline Steel casing & 7850 & 200.0 & 0.33 \\
\hline Ag-Epoxy adhesive & 1700 & 2.7 & 0.45 \\
\hline Brass backing & 8530 & 113.4 & 0.33 \\
\hline Wear plate & 3965 & 400.0 & 0.22 \\
\hline $\begin{array}{l}\text { Lead-Zirconate-Titanate } \\
\text { (PZT)-5A }\end{array}$ & 7750 & $\begin{array}{l}\mathrm{C}_{11}=\mathrm{C}_{22}=120.3 \\
\mathrm{C}_{12}=75.2 \\
\mathrm{C}_{13}=\mathrm{C}_{23}=75.1 \\
\mathrm{C}_{33}=110.9 \\
\mathrm{C}_{44}=\mathrm{C}_{55}=21.1 \\
\mathrm{C}_{66}=22.6\end{array}$ & - \\
\hline
\end{tabular}

Tab. 1. Structural material properties used in this study.

\begin{tabular}{|l|l|l|}
\hline Name & Electrical permittivity & Piezoelectric constants $\left[\mathbf{C} / \mathbf{m}^{2}\right]$ \\
\hline Lead-Zirconate- & $\xi_{11}=919.1$ & $\mathrm{~S}_{31}=\mathrm{S}_{32}=-5.4$ \\
Titanate (PZT)-5A & $\xi_{22}=919.1$ & $\mathrm{~S}_{33}=15.8$ \\
& $\xi_{33}=826.6$ & $\mathrm{~S}_{24}=\mathrm{S}_{15}=12.3$ \\
\hline
\end{tabular}

Tab. 2. Electrical material properties used in this study.

\section{Experimental verification}

Extensive validation of the presented numerical approach has been carried out in previous research [9], [10], [15]. As a brief review of these contributions, we present two exemplary results of these investigations. On the experimental side, signals of pencil lead breaks were detected by a Polytec model OFV-5000 Laservibrometer with a model DD-900 displacement decoder in triggered mode at $10 \mathrm{MHz}$ sampling rate. The system allows detection of the outof-plane displacement component directly, if placed at orthonormal incidence to the plate surface. All signals were filtered using an analog $6^{\text {th }}$ order Butterworth bandpass ranging from $20 \mathrm{kHz}$ to $1 \mathrm{MHz}$. This experimental out-of-plane signal is compared against the calculated outof-plane surface displacement as obtained in the Lamb wave configuration (cf. Fig. 1d) without presence of the sensor. Fig. 4-a shows a comparison of both signals, indicating a high level of 
quantitative agreement, although the Laservibrometer data quality suffers from its signal-tonoise ratio.

In another study [9] we experimentally assessed the Lamb wave configuration for the conical sensor type used in this study. A comparison of the experimentally measured signal and the electrical voltage signal as calculated using our modeling approach is shown in Fig. 4-b. Again, a high degree of quantitative agreement is found concerning the signal amplitudes and the signal phase of both signals. For the study in [9] a distance of $250 \mathrm{~mm}$ between pencil lead break source and sensor was chosen, thus the signal appearance and the duration selected differs from Fig. 4-a.
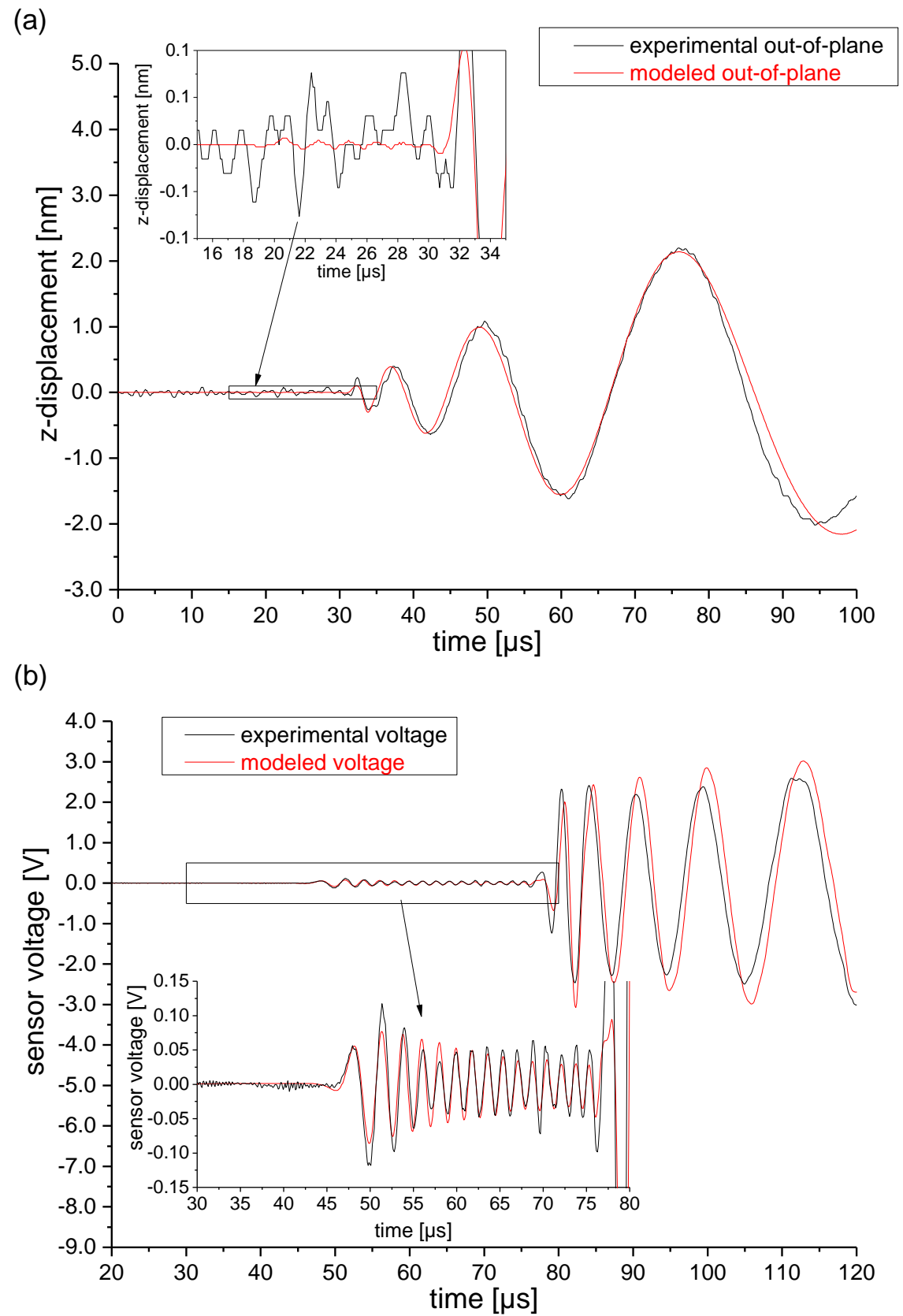

Fig. 4. Comparison of out-of-plane displacement in Lamb wave configuration as detected by Laservibrometer and as modeled (a) and corresponding comparison of experimental $\mathrm{AE}$ sensor signal vs. modeled AE sensor signal (b). 
As only material parameters and the propagation medium geometry are parameterized in this study, the overall modeling approach retains unchanged. We thus consider our approach to be experimentally validated for the further analysis within this study.

\section{Results and discussion}

In the following, we present and discuss the findings of this study in view of three aspects relevant to practical $A E$ sensor calibration approaches. Thus, we consider the influence of the wave propagation type, the propagation medium material and the sensor design. In addition, the use of Lamb waves for AE sensor calibration is reviewed.

\subsection{Wave propagation type}

A first overview is presented to understand the differences of wave propagation in the four principle arrangements investigated. To this end, we chose the specific case of steel as propagation medium and the conical sensor type in the following.

As seen in Figs. 5, 6, 7 and 8 the type of received AE signal is significantly different for each configuration. Each figure shows the surface normal displacement component (z-component) at the center-point of the AE sensors contact surface without the AE sensor being in place (as used in Standard Test Method ASTM E1106) and the corresponding electrical voltage signal as computed for the AE sensor model in place.

For the bulk wave propagation seen in Fig. 5, there is an arrival of the longitudinal wave at $17.0 \mu \mathrm{s}$, followed by an arrival of the transversal wave at $31.7 \mu \mathrm{s}$. The computed zdisplacement coincides well with the analytical results for surface displacement due to stepfunction loading as published in [4], [14]. The conical sensor type, despite being almost flatwith-frequency, does not exactly convert the z-displacement into an electrical voltage signal. The $410 \mathrm{~Hz}$ high-pass of the attached circuitry limits the bandwidth at the lower end of the frequency range and the small backing does not provide sufficient mass to convert low frequency signals without causing additional losses (i.e. frequency range below $100 \mathrm{kHz}$ ). This relationship has been explored concisely in [19]. The signal voltage polarity appears inverted relative to the z-displacement, which is caused by the definition of the PZT polarization direction, the choice of ground and signal position and the orientation of the z-axis. At $51 \mu \mathrm{s}$ and beyond, reflections of the longitudinal wave appear at the sensor position. These originate from the direct reflection at top surface, then bottom surface and back to the sensor positon (i.e. $300 \mathrm{~mm}$ distance of propagation). To avoid any influence in obtaining the sensitivity curves, signals were truncated at time values according to table 3 (as illustrated in Fig. 5) to eliminate any secondary arrivals of longitudinal or transversal waves. 


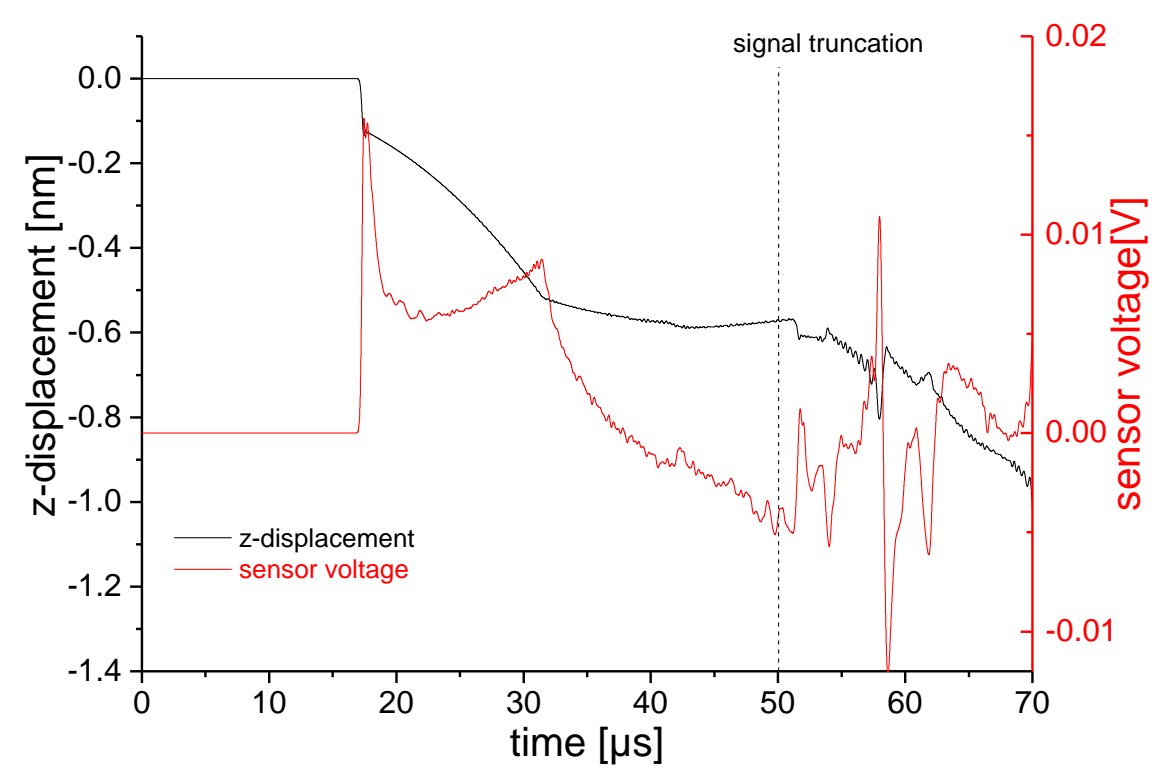

Fig. 5. Comparison of surface normal displacement component and AE sensor signal voltage for bulk wave propagation.

In Fig. 6, the computed normal displacement for rod waves is shown with the AE sensor signal superimposed. The dominant component of the z-displacement is the movement of the rod as consequence of the applied force, resulting in an almost constant drift, as the rod is not pinned in the z-direction. Besides these low frequency contents, several superimposed guided wave modes are excited. These manifest themselves in high-frequency oscillations in the zdisplacement and result in even more pronounced oscillations for the sensor voltage signal.

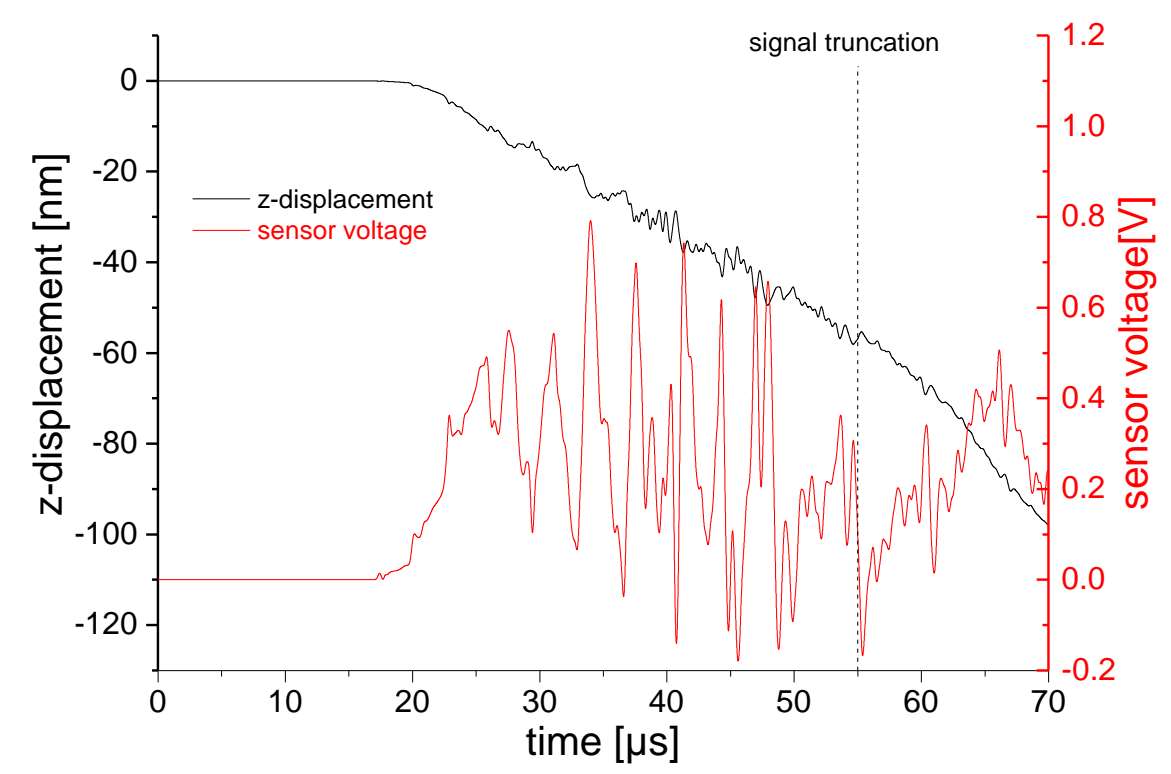

Fig. 6. Comparison of surface normal displacement component and AE sensor signal voltage for rod wave propagation.

The computation results for the Rayleigh wave propagation are shown in Fig. 7. The obtained $\mathrm{z}$-displacement in this configuration shows a distinct arrival of the longitudinal wave at $17.0 \mu \mathrm{s}$ followed by the arrival of the Rayleigh wave at $34.0 \mu \mathrm{s}$. These values correspond with analytical results (cf. e.g. [20]). After $58 \mu \mathrm{s}$, first reflections of the longitudinal wave from the 
bottom part of the block arrive at the sensor location. Accordingly, signals were truncated prior to further analysis at time values listed in table 3 to avoid the influence of significant signal reflections.

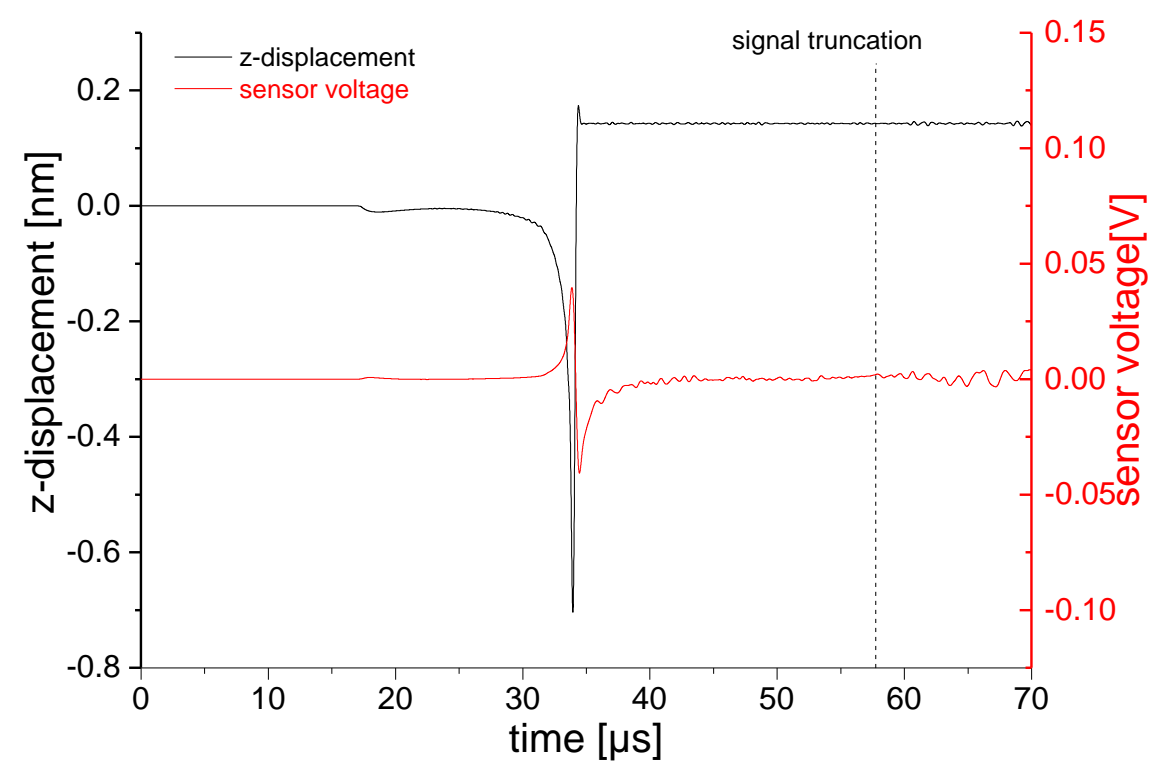

Fig. 7. Comparison of surface normal displacement component and AE sensor signal voltage for Rayleigh wave propagation.

For the plate as propagation medium, guided waves are generated in the form of Lamb waves. The resulting z-displacement for the case of the $3 \mathrm{~mm}$ thickness plate is shown superimposed with the sensor voltage signal in Fig. 8. The first arriving wave mode $S_{0}$ arrives at $18.3 \mu \mathrm{s}$, it is followed by the $S_{1}$ mode arriving at $25.7 \mu \mathrm{s}$. The largest discrepancy between z-displacement and sensor voltage is seen for the lower frequency dominated $A_{0}$ mode arriving at $30.9 \mu \mathrm{s}$. Based on the chosen size of the plate, first reflections of the $S_{0}$ mode are incident at the sensor position at $73.2 \mu \mathrm{s}$. Thus, the superimposed high frequency oscillations seen in Fig. 8 are not caused by reflections, but originate from the contributions of the primary arrival of $\mathrm{S}_{0}$ mode and the $S_{1}$ mode. To avoid including reflections from the edge of the plate the signals were terminated at time values listed in table 3 before further analysis. 


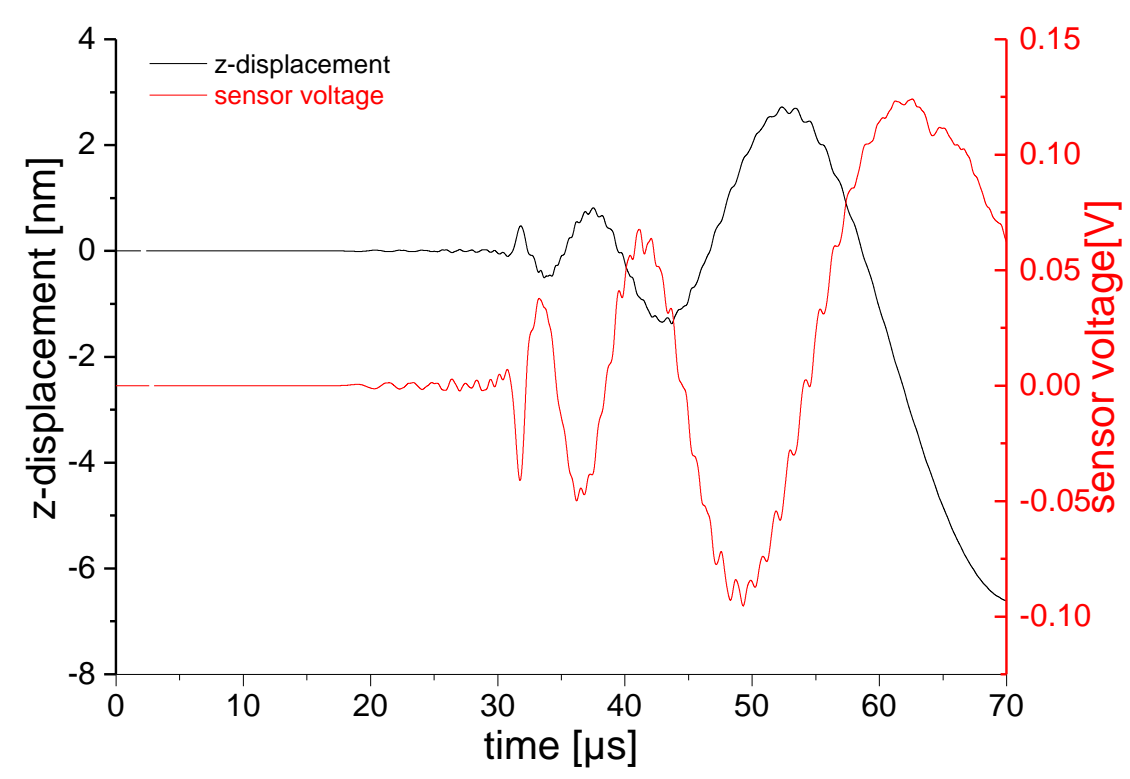

Fig. 8. Comparison of surface normal displacement component and AE sensor signal voltage for Lamb wave propagation.

Following the approach outlined in [9] it is feasible to obtain AE sensor sensitivity curves by relating the $A E$ sensor signal voltage to the surface normal displacement. This division is done in the frequency domain after Fast-Fourier-Transformation (FFT) of each signal. All signals are truncated, before arrival of no or significant edge and boundary reflections as listed in table 3. Then a linear curve is subtracted from the signals (displacement and voltage) to obtain zero values at the beginning and the end of the truncated time range and zero-padded to reach 32768 points. The linear curve is used for the case of unequal initial and final values, so that spurious frequencies are not introduced by the periodicity assumption of the FFT algorithm. Reference [21] demonstrates that a modification with a linear slope does not change the frequency content and successfully avoids the introduction of such spurious frequencies. All FFTs are done using the Cooley-Tukey algorithm in base-2 form with a rectangular window function to yield a frequency step of $6.104 \mathrm{kHz}$. To facilitate visibility of the relevant differences the calculated sensitivity curves were smoothed using a $2^{\text {nd }}$ order Savitzky-Golay filtering with 20 points.

\begin{tabular}{|l|l|l|l|l|}
\hline Material & Bulk wave & Rayleigh wave & Rod wave & Lamb wave \\
\hline Steel & 50 & 68 & 55 & 70 \\
\hline Aluminum & 47 & 65 & 55 & 70 \\
\hline PMMA & 100 & 130 & 130 & 140 \\
\hline
\end{tabular}

Tab. 3. Signal evaluation length to avoid superimposed reflections for the different configurations (all values are in microseconds).

The comparison of the four types of wave propagation configurations studied is shown in Fig. 9-a for a steel medium. Despite the different geometric choices, there is some resemblance of the sensitivity curves. Especially for steel as propagation medium, the four different curves agree reasonably well in the frequency range less than $500 \mathrm{kHz}$ (cf. Fig. 9-a). For the curves obtained for the rod and for the plate there are some larger fluctuations in the curves, which are likely due to the formation of guided wave modes and their difference in interaction with 
the attached sensor (see section 3.4). The observed overlap of different configurations seen for the steel cases is not a general trend as exemplified by the same analysis result for aluminum as propagation medium (cf. Fig. 9-b). Such overlap was not observed for PMMA either (cf. Fig. 8-c). Thus, this important material aspect is discussed further in the next section.

(a)

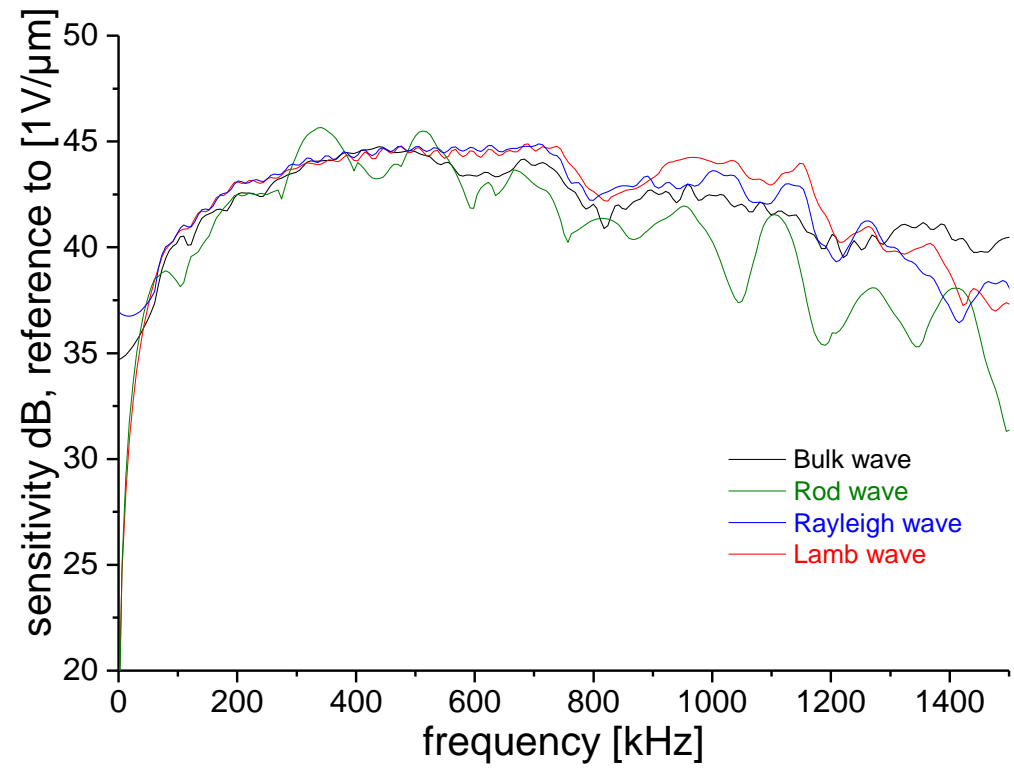

(b)

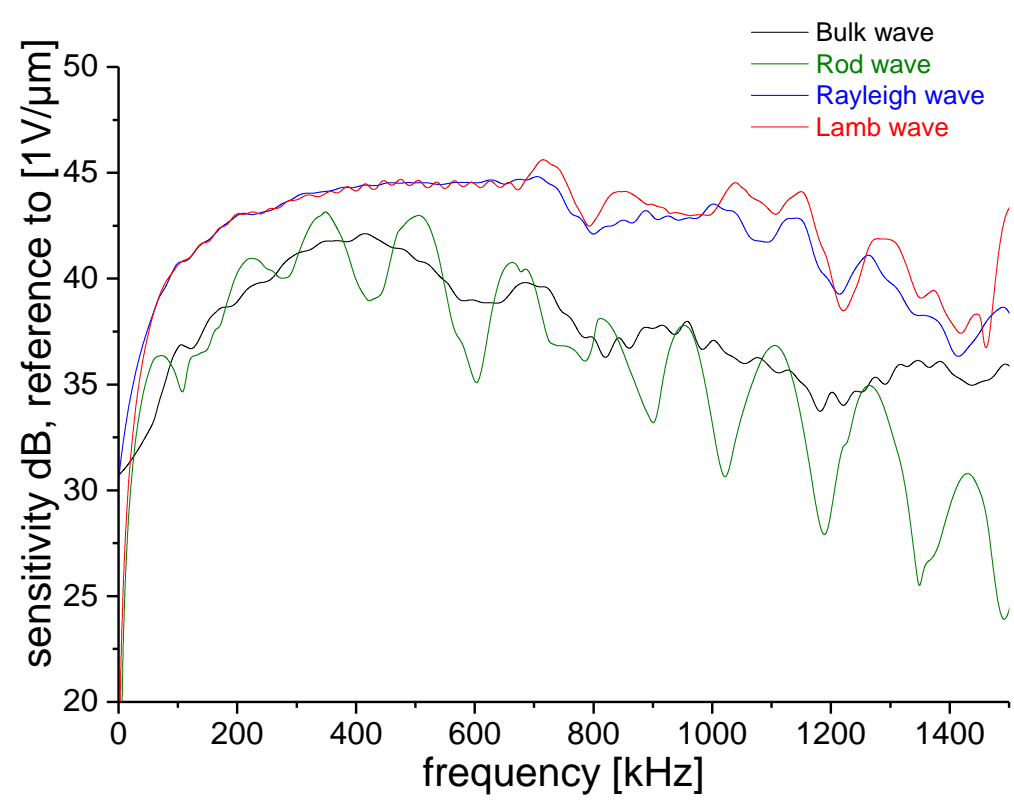


(c)

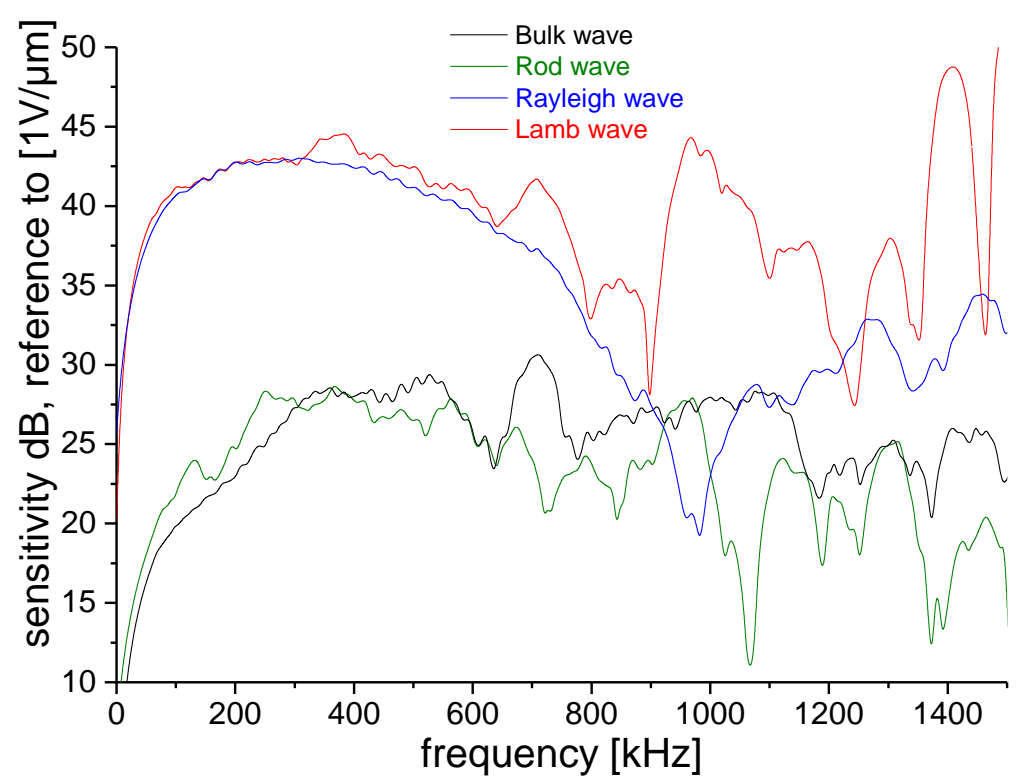

Fig. 9. Comparison of $A E$ sensor sensitivity curves for the four wave propagation types in steel (a), aluminum (b) and PMMA (c).

\subsection{Propagation medium material}

As the second factor investigated, the models were evaluated for three different material property sets for the propagation medium for the bulk and Rayleigh wave cases. The typical choices discussed in the context of standards and proposed modifications are steel alloys, aluminum alloys and polymers (specifically PMMA). As has been pointed out in literature several times [10], [22], [23], the propagation medium used for sensor calibration will not only affect the character of the signals due to changes in the sound velocity, but will also inherently change the sensor response due to the change in acoustic impedance relative to the sensor(s) face or wear plate face [10], [23]. Hence, there is no expectation for a unique sensitivity curve for an AE sensor, regardless of the material the sensor is mounted on. This difference is elucidated by taking the propagation geometries above and replacing the elastic properties of the propagation medium. For PMMA the duration was increased to the values in table 3 to allow full arrival of the bulk waves and the Rayleigh waves (without superimposed reflections). For the bulk wave configuration, the sensor sensitivity in Fig. 10-a shows a systematic change in sensitivity as function of the propagation medium. In this configuration, the wave arrives as plane wave front from within the propagation medium and needs to pass the interface to the sensor. Based on the acoustic impedance mismatch between the PZT cone and the propagation medium the transmission and reflection coefficients should change. For orthogonal incidence of longitudinal waves, the calculated decay (impedance mismatch) in $\mathrm{dB}$ is noted in Fig. 10-a by the length of the vertical arrows. Despite some frequency dependency, the analytically calculated drop in sensitivity of $4.6 \mathrm{~dB}$ and $7.5 \mathrm{~dB}$ agrees reasonably well with the modeling results. Frequency ranges with strong "resonances" seem to exhibit stronger or weaker decays than analytically calculated.

For the Rayleigh wave configuration, such simple considerations as orthogonal incidence are no longer valid and aperture effects need to be taken into account. In this context, the huge drop of sensitivity of the PMMA case at around $980 \mathrm{kHz}$ is caused by the aperture of the conical sensor element. Compared to the Rayleigh wave velocities of steel and aluminum, the reduction of propagation speed of the PMMA causes the propagation wavelength to match the aperture of the conical sensor and causes a pronounced reduction of sensitivity in this 
frequency range. Similar effects are not observed for steel and aluminum, as the relevant wavelengths stay above $1.5 \mathrm{MHz}$. In other studies considering Rayleigh wave based calibration [24], theagreement in sensor sensitivity curves was reported for steel and aluminum as well. Also, a decrease of about $15 \mathrm{~dB}$ sensitivity between these materials and PMMA was reported. This agrees with the tendency of our numerical results.

(a)

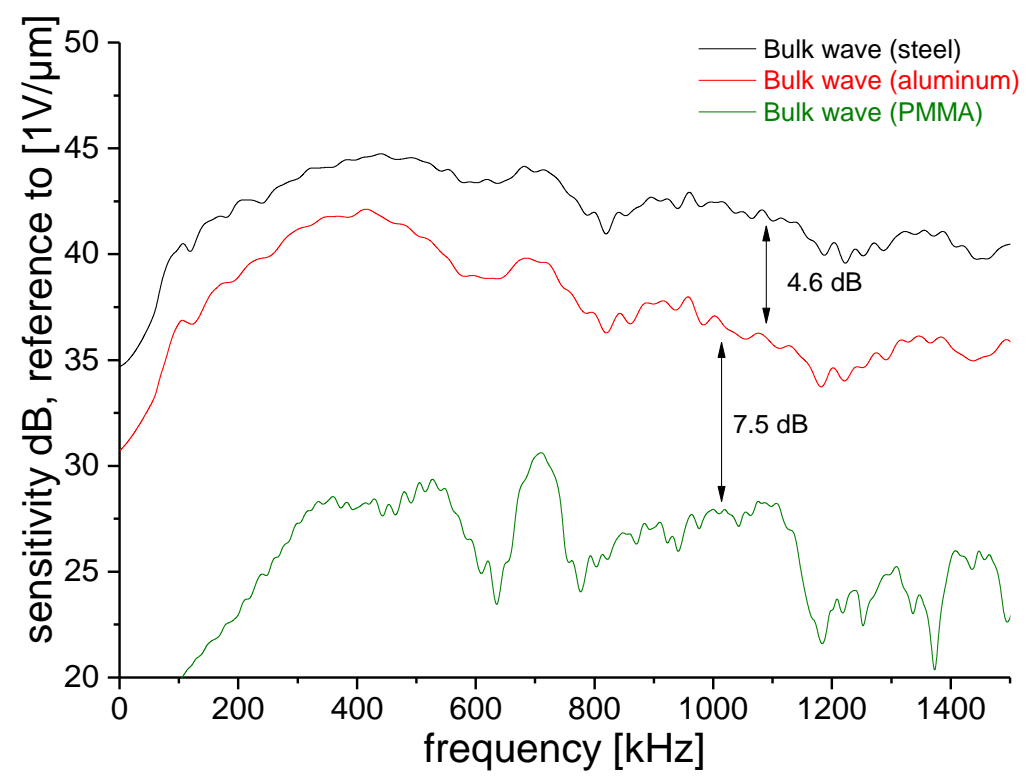

(b)

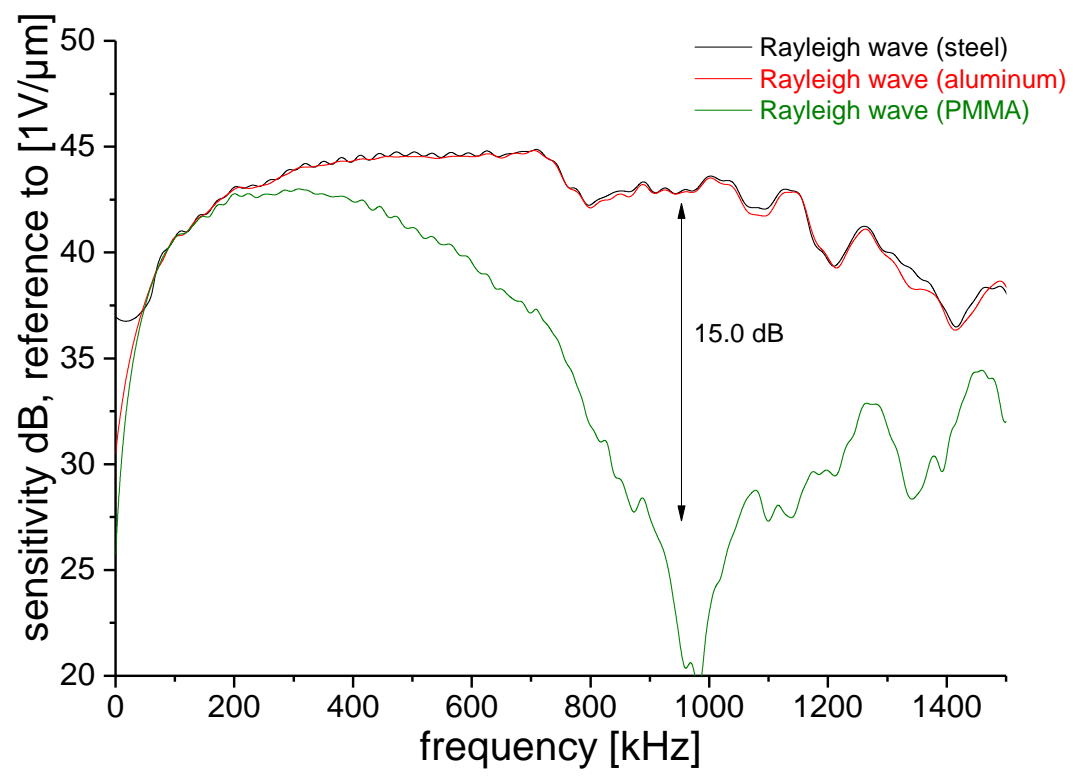

Fig. 10. Comparison of AE sensor sensitivity curves for bulk wave (a) and Rayleigh wave (b) configuration and material properties of steel, aluminum and PMMA.

\subsection{Sensor design}

Keeping steel as the propagation medium, the next effect to be investigated is the sensor design. To this end, we compare the two principle designs outlined in Fig. 2 . The results so far are obtained for a conical type sensor, which is considered to be reasonably flat-withfrequency. Despite the resonances seen in the Figs. 9 and 10, this terminology is shown to be appropriate when compared to a typical disc sensor design. Plotted in Fig. 11-a is the time 
domain signal response of both sensor types from the bulk wave arrivals. Here, the conical sensor follows the theoretically expected surface displacement much closer than the disc sensor. The latter shows pronounced reverberations at a characteristic frequency around $113 \mathrm{kHz}$. According to a resonance analysis of our disc sensor model, this frequency relates to the thickness resonance of the $5 \mathrm{~mm}$ disc in the presence of the sensor casing. By obtaining the frequency sensitivity as explained in section 3.1, the difference is even easier to observe. In the direct sensitivity comparison (Fig. 11-b), the conical sensor is reasonably flat-withfrequency, while the disc sensor does show a strong resonance at $113 \mathrm{kHz}$ followed by multiple other higher-order resonances. The sensitivity of the disc sensor appears up to $30 \mathrm{~dB}$ higher than the conical sensor, although the sensitivity falls of by $30 \mathrm{~dB}$ in the range up to $1400 \mathrm{kHz}$. The occurrence of the resonance at $113 \mathrm{kHz}$ is persistent for the case studies of sensitivity with Rayleigh waves (cf. Fig. 11-c) and Lamb waves (cf. Fig. 11-d). Some of the drops of sensitivity of the Rayleigh wave case and the Lamb wave case are caused by the aperture effect, as the extinction frequencies are moved to much lower frequencies, compared to the conical sensor (first extinction frequency at $185 \mathrm{kHz}$ for the $16 \mathrm{~mm}$ disc diameter). Also, the basic tendency of about $30 \mathrm{~dB}$ sensitivity fall-off in the range between $113 \mathrm{kHz}$ and $1400 \mathrm{kHz}$ is consistent for the disc sensor in all three cases. However, the overall sensitivity of the disc sensor seems to change depending on the incident wave type. The highest sensitivity is found for the bulk wave case, which is due to the direct plane wave front arrival from below the sensor. This incidence angle is beneficial to convert the thickness deformation (z-axis) of the disc into an electric voltage signal. For the Rayleigh wave case and the Lamb wave case, the wave propagation direction is orthogonal to the thickness direction of the disc. Due to the PZT-polarization direction along the $z$-axis, disc deformation components in $\mathrm{x}$ - and $\mathrm{y}$-direction, as well as stimulated torque deformations are not equally converted to electrical signals as the deformation along the z-axis. This influence of the wave incidence angle will be of particular importance when detecting multiple guided wave modes as discussed in section 3.4.4.

(a)

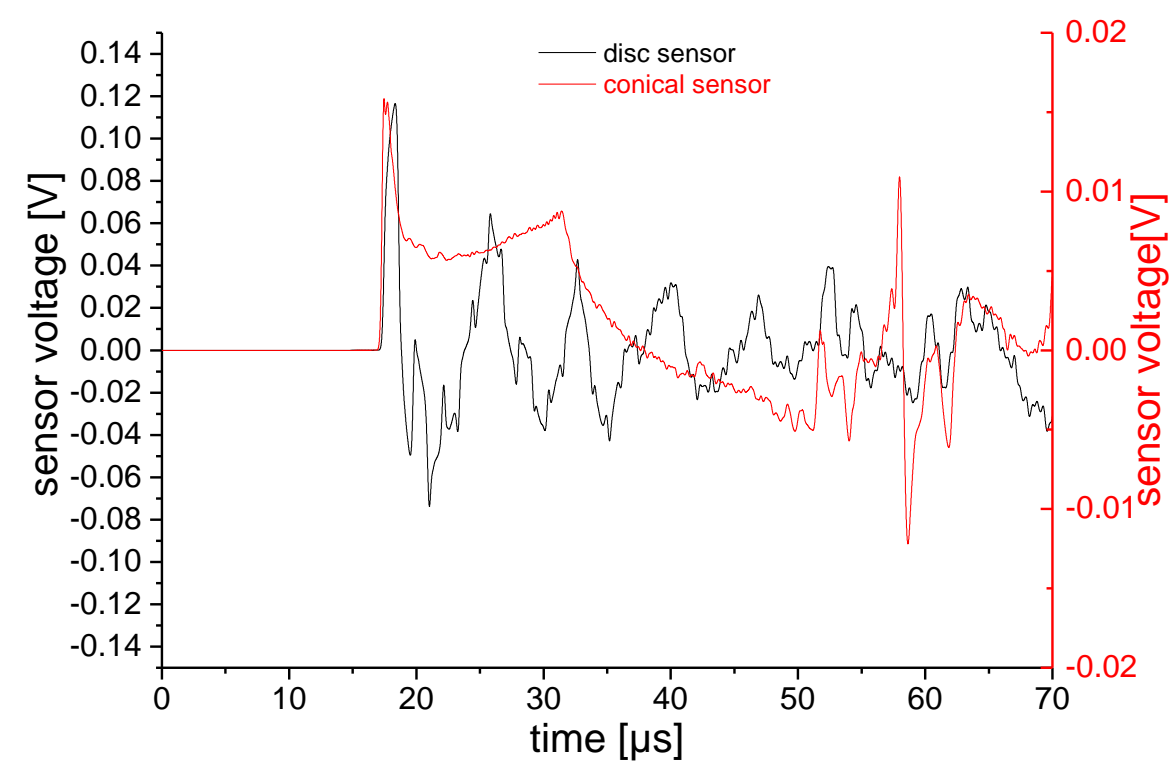


(b)

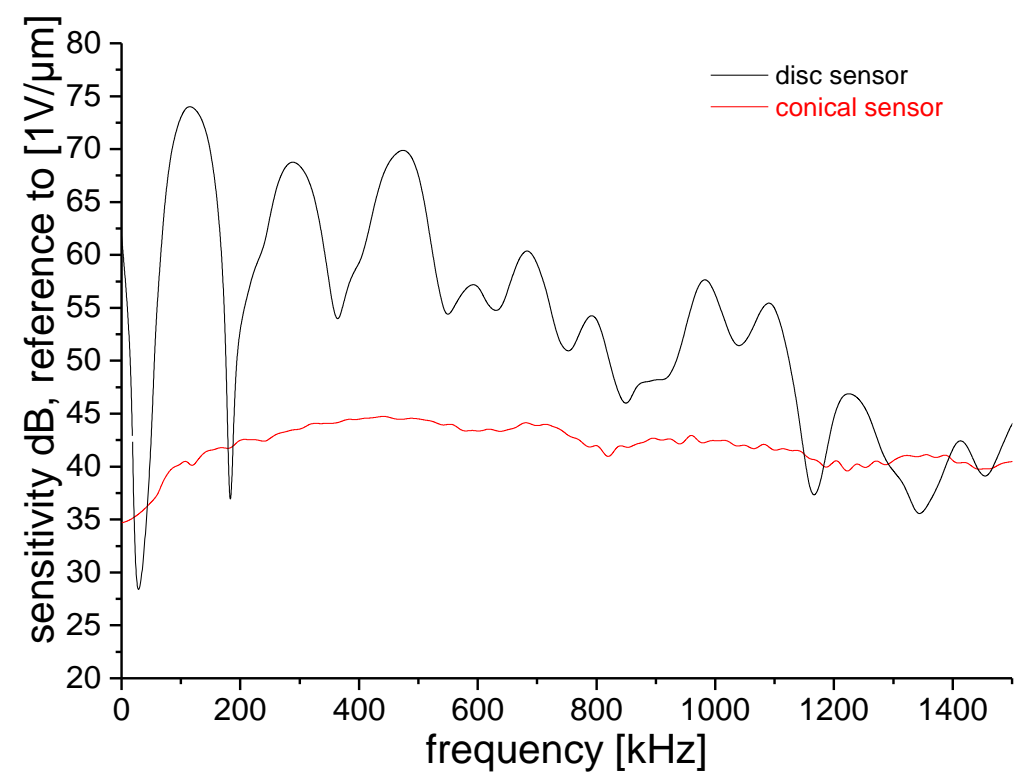

(c)

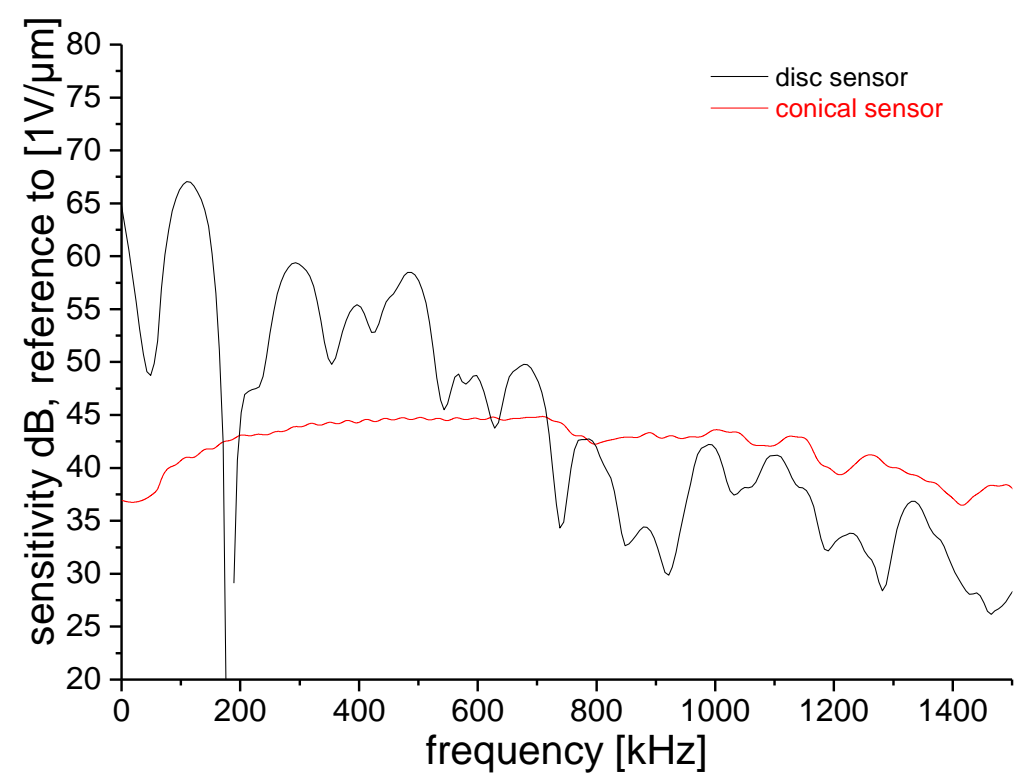


(d)

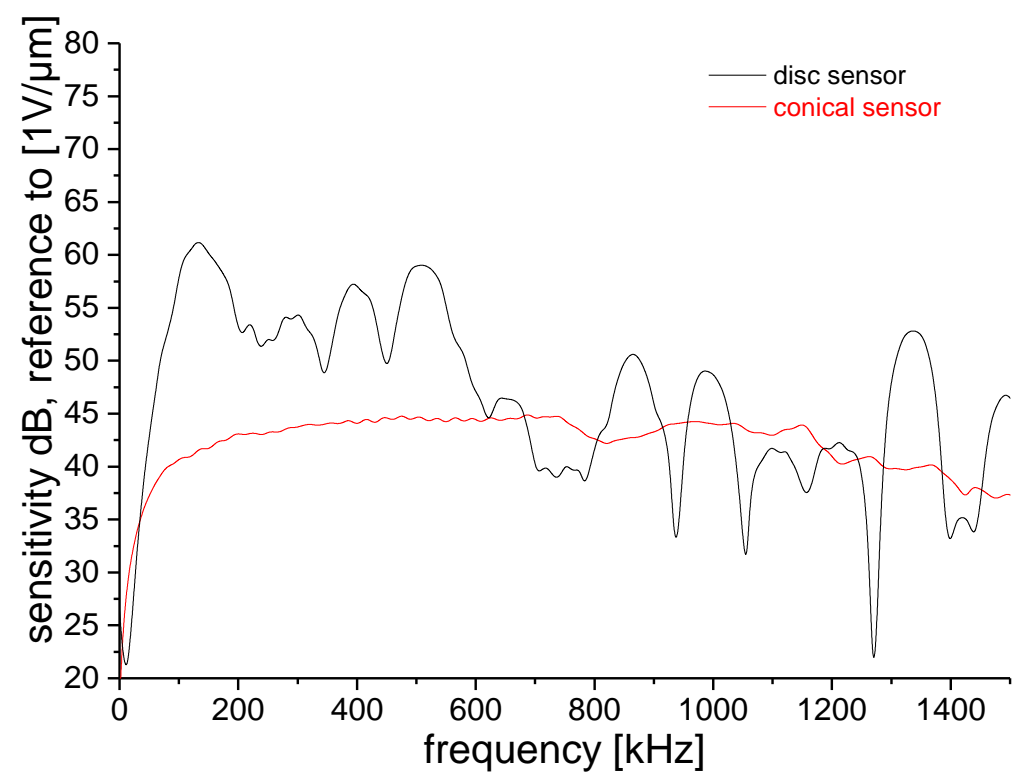

Fig. 11. Comparison of AE sensor response (two sensor geometries see Figs. 2 and 3, in particular apertures) for bulk wave configuration and steel medium time domain (a) and corresponding frequency sensitivity spectrum (b). Comparison of sensitivity curves for Rayleigh wave configuration (c) and Lamb wave configuration (d) for steel medium.

\subsection{Use of Lamb waves}

Based on the close match of sensitivity curves of Lamb waves and Rayleigh waves for all the cases investigated it seems tentative to propose the use of plates with guided waves to obtain sensor sensitivity curves in spite of the potential benefits listed next. First, many applications use $A E$ sensors on plate-like structures, so that their sensitivity relative to guided wave modes would be the relevant factor in choosing a sensor type. Second, the handling of plate materials instead of large solid blocks is much easier due to their weight. In addition, given the relatively low financial investment for different plate materials, this choice would facilitate calibration measurements for different materials. In the following, we discuss several key experimental factors relevant for Lamb wave based calibration.

\subsubsection{Thickness of the plate}

Similar to that for the other propagation media, the geometry of the plate needs to be considered. The edge length of the plate is selected based on usual considerations to avoid incident reflections during the duration of the measurement pulse, which could cause artificial structures within the signals [25], [26]. This consideration leads to typical plate sizes in the range of $1-2 \mathrm{~m}$. In addition, the thickness of the plate deserves some additional attention. For the same test source and constant bandwidth, the thickness of the plate is responsible for the number and type of Lamb wave modes. For a typical range, we investigated a steel plate thickness of $1.5 \mathrm{~mm}, 3.0 \mathrm{~mm}$ and $6.0 \mathrm{~mm}$. The computed conical sensor signals are shown in Fig. 12. In addition, the smoothed Pseudo-Wigner-Ville distribution was calculated for each signal and the Lamb wave dispersion curves were superimposed to allow mode identification. The resulting sensitivity spectra are shown in Fig. 13-a. Overall, there is some scatter in between the curves, which is due to the differences in the detected Lamb wave modes. In all three cases, the dominant wave mode is the $A_{0}$. However, with increasing thickness, additional 
contributions of $S_{0}, S_{1}$ and $A_{1}$ arise (cf. Fig. 12-d for Pseudo-Wigner-Ville distribution after $100 \mathrm{kHz}$ high-pass of signal of Fig. 12-c). These Lamb wave modes propagate predominantly at particular frequencies, thus causing preferential transmission of the wave's bandwidth close to these frequencies (cf. Ao mode close to $50 \mathrm{kHz}$ ). In addition, the choice of the source with a force acting in z-direction causes preferential excitation of the antisymmetric wave modes.

Nevertheless, the discrepancy between the three sensitivity curves of Fig. 13-a is less prominent than most of the other factors investigated herein. As the conical sensor design is a point-contact sensor with preferential sensitivity to the out-of-plane displacement motion of the various guided wave modes, this situation might easily change when turning to a disc sensor with extended aperture. Repeating the study with the disc sensor in place, the sensitivity curves of Fig. 13-b are obtained. At some frequencies, deviations between curves of different plate thickness of up to $20 \mathrm{~dB}$ are observed. Best agreement is found for the first resonance peak at $113 \mathrm{kHz}$, which is in the range of the strongest excitation of the $A_{0}$ mode. As for the thinner plates the frequency range above $200 \mathrm{kHz}$ is barely stimulated, this may partially be responsible for the lack of agreement between curves. Another factor, which might add to the discrepancy between the sensitivity curves, is the additional change in intensity of the different guided wave modes, which is elaborated in sections 3.4.3 and 3.4.4. Thus, it seems that the choice of plate thickness is of particular importance for the calibration procedure. However, there is an expectation of reduced influence for thicker plate sizes than investigated as more and more guided wave modes are compressed into the frequency range of interest. This would cause a more uniform transmission characteristic, thus narrowing gaps of poor signal-to-noise ratio.

(a)
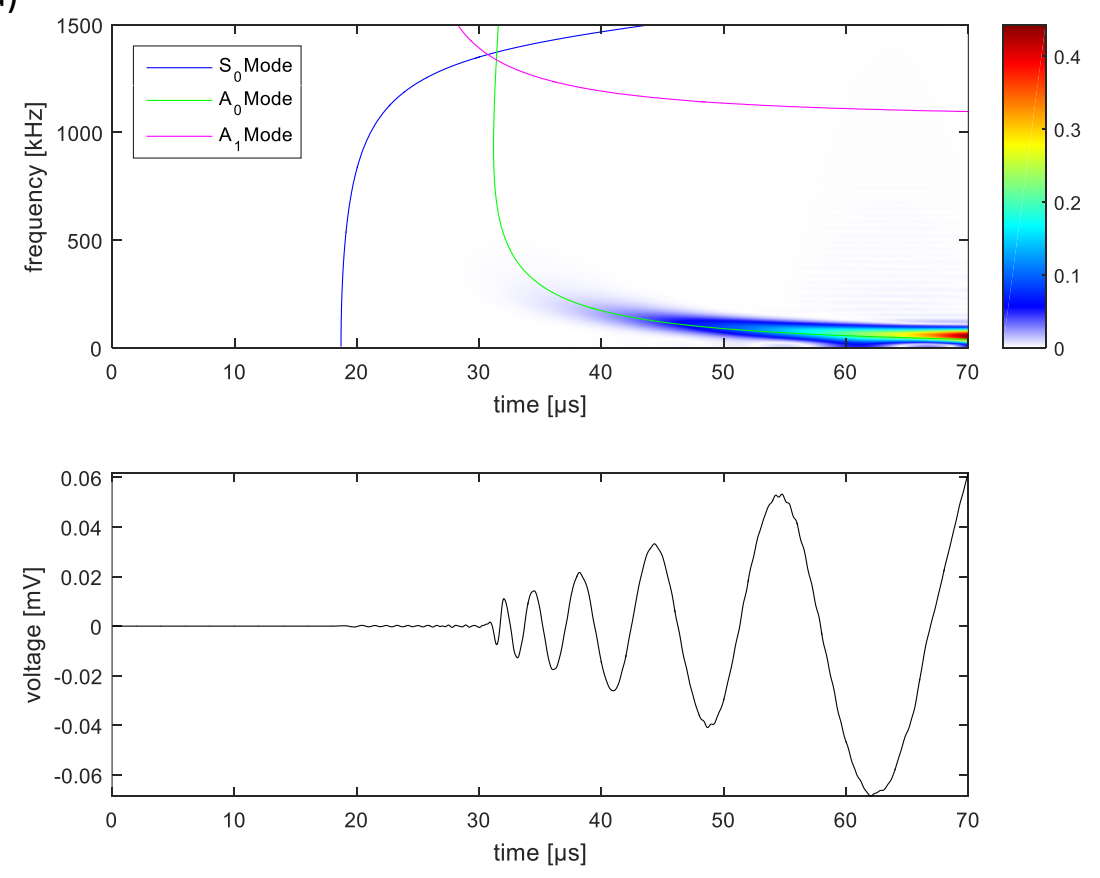
(b)
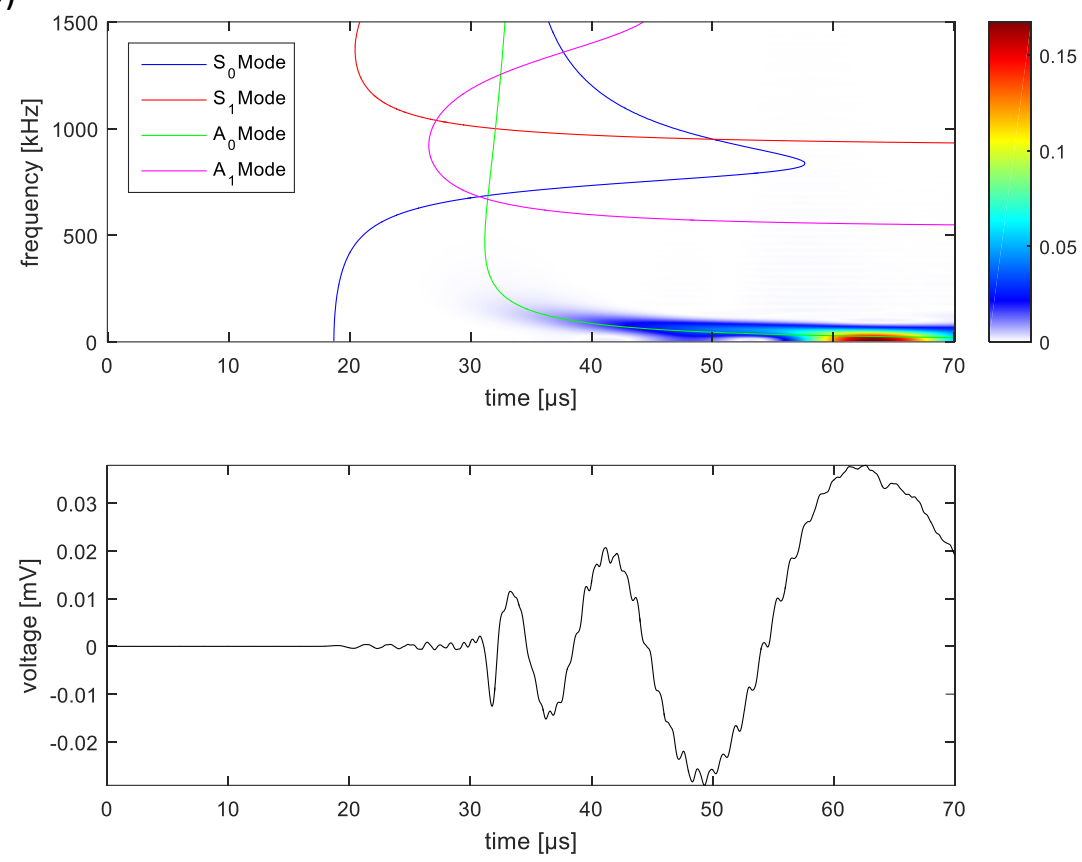

(c)
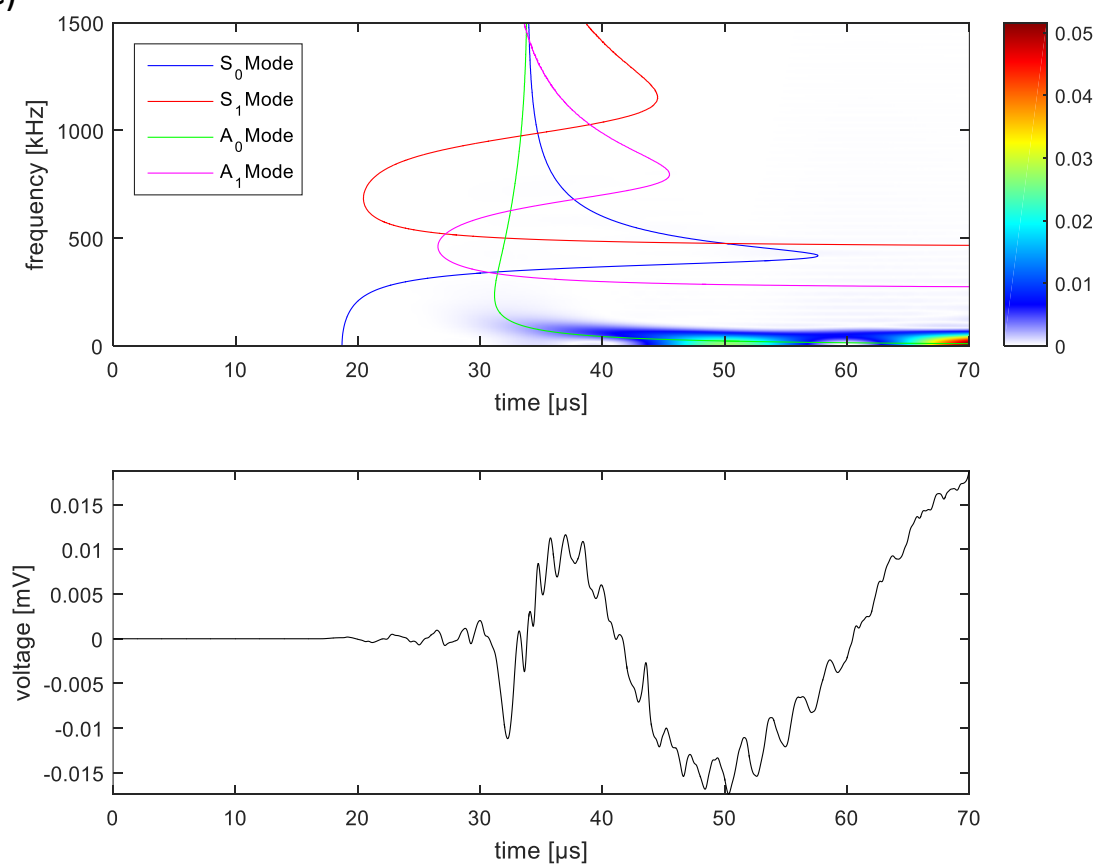
(d)
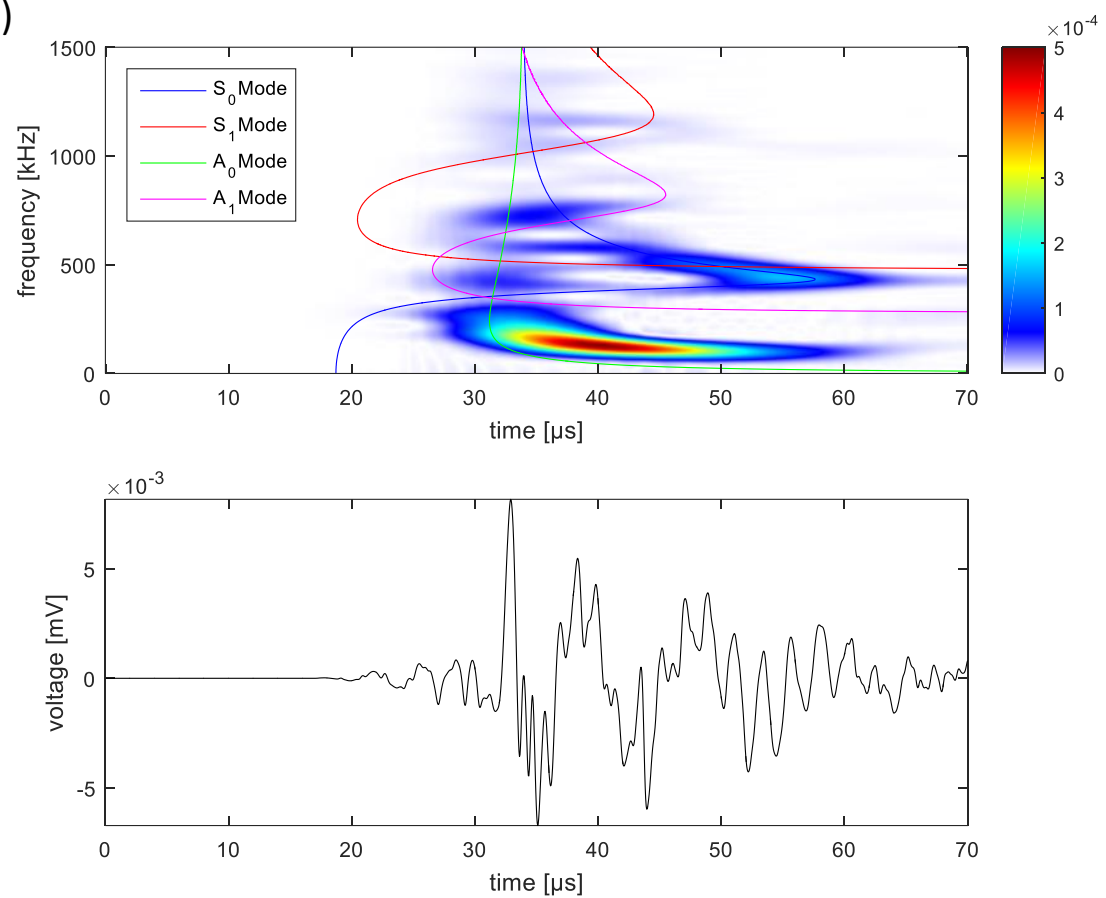

Fig. 12. Comparison of conical sensor signals detected in steel plate with thickness of $1.5 \mathrm{~mm}$ (a), $3.0 \mathrm{~mm}$ (b) and $6.0 \mathrm{~mm}$ (c) with corresponding smoothed Pseudo-Wigner-Ville distribution and superimposed dispersion curves. Signal of plate thickness $6.0 \mathrm{~mm}$ after $100 \mathrm{kHz}$ high-pass filtering to enhance visibility of higher order modes (d).

(a)

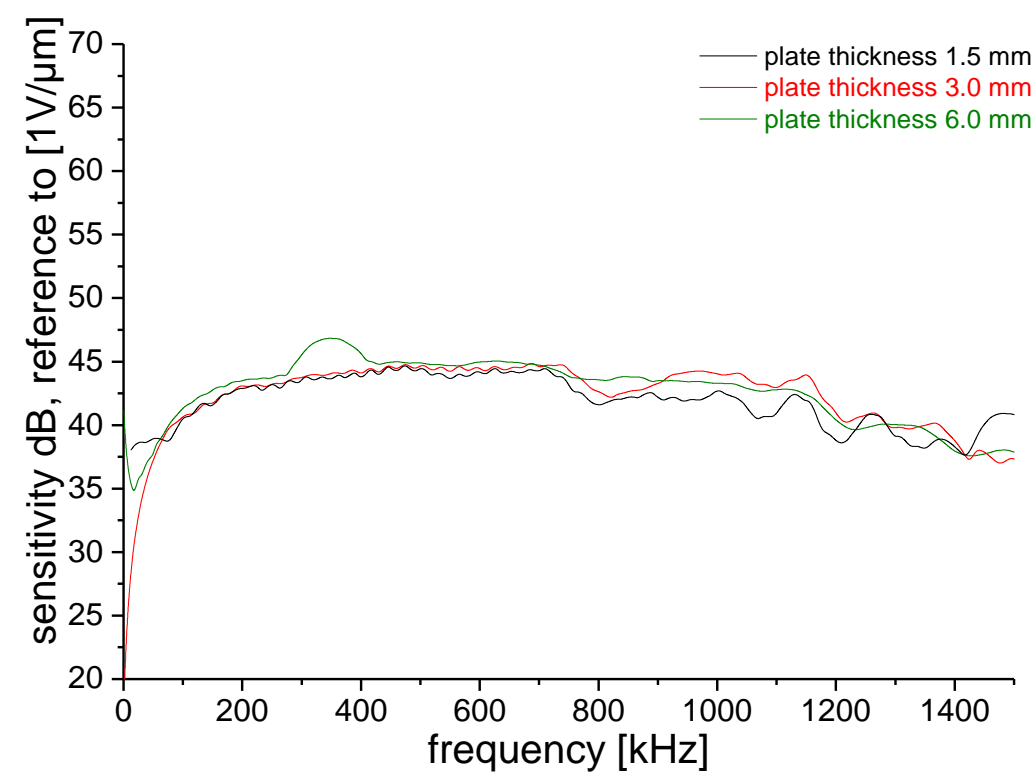


(b)

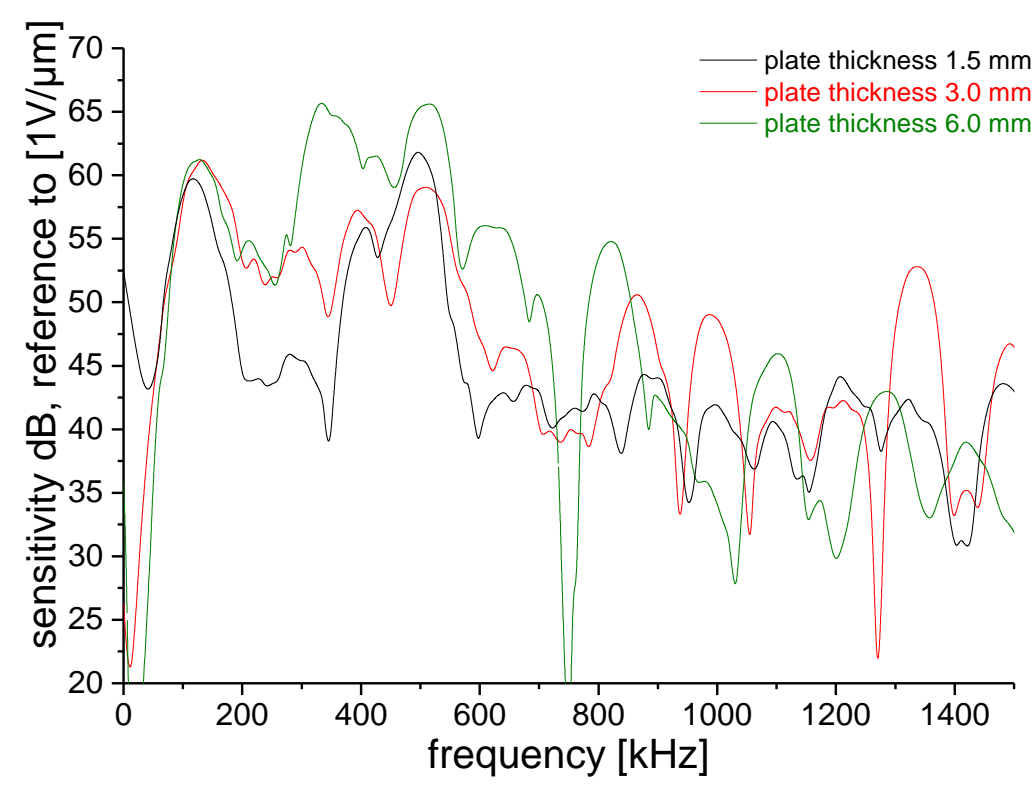

Fig. 13. Comparison of conical sensor sensitivity (a) and disc sensor (b) for steel plate with thickness of $1.5 \mathrm{~mm}, 3.0 \mathrm{~mm}$ and $6.0 \mathrm{~mm}$.

\subsubsection{Propagation distance}

Another obvious parameter that needs specific attention is the distance between the source and sensor. This parameter is not only crucial for Lamb waves, but also the other geometries considered herein. However, the formation of distinct Lamb wave modes requires a certain propagation distance, which has been estimated between 5 times the thickness [27] and 13 times the thickness [28]. Moreover, due to dispersion effects, the propagation distance and the required evaluation length are intimately related (cf. also section 3.4.3).

In the modeled scenarios, the lowest propagation distance was chosen as 8.33 times the plate thickness of $3 \mathrm{~mm}$, i.e. at $25 \mathrm{~mm}$ to the source position, and the maximum distance was 66 times the plate thickness. For these models, displacement signals and sensor signals were evaluated at the designated distances, resulting in the waves shown in Fig. 14. To avoid other influences, all signals were evaluated for $83 \mu$ s total duration (see 3.4 .3 for this influence), starting with equal offset to the first arrival of the $S_{0}$ mode (cf. Fig. 14). The corresponding sensitivity spectra are shown in Fig. 15. For the shorter two distances $(25 \mathrm{~mm}$ and $50 \mathrm{~mm})$ the spectra differ in various aspects from the spectra computed for the larger two distances $(100 \mathrm{~mm}$ and $200 \mathrm{~mm}$ ). The $25 \mathrm{~mm}$ case exhibits a lower overall sensitivity in the frequency range up to $800 \mathrm{kHz}$. The $50 \mathrm{~mm}$ case shows some pronounced "resonances" above $600 \mathrm{kHz}$. We attribute this behavior to the dispersion effect also seen in the waves of Fig. 14. For the shorter two distances there are still longer wavelengths (from $A_{0}$ mode) being accounted for in the sensitivity spectra calculation. These lowest frequencies are no longer considered in the calculation of the $100 \mathrm{~mm}$ case and the $200 \mathrm{~mm}$ case as the chosen $83 \mu$ s does not include these wavelengths that due to dispersion would arrive later. The relevance of this change in the detected and analyzed frequencies will be discussed in more detail in the sections 3.4.3 and 3.4.4. For this part of the study, it seems sufficient to consider distances, which are significantly far apart from the source position, i.e. there is no significant difference between the $100 \mathrm{~mm}$ case and the $200 \mathrm{~mm}$ case. 


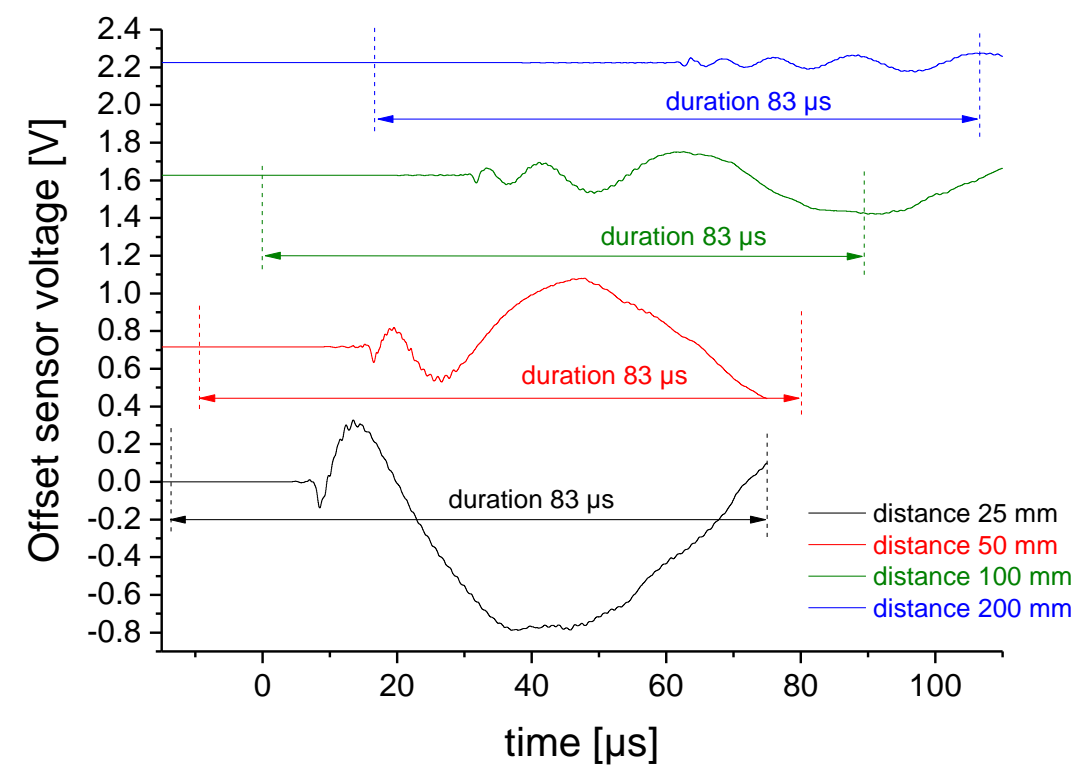

Fig. 14. Comparison of conical sensor signals on steel plate $(3 \mathrm{~mm})$ for different distances between source and sensor. Start of source excitation at $t=0 \mu \mathrm{s}, 83 \mu \mathrm{s}$ duration were reached by zero padding / truncation to $17 \mu$ s before initial arrival of So mode.

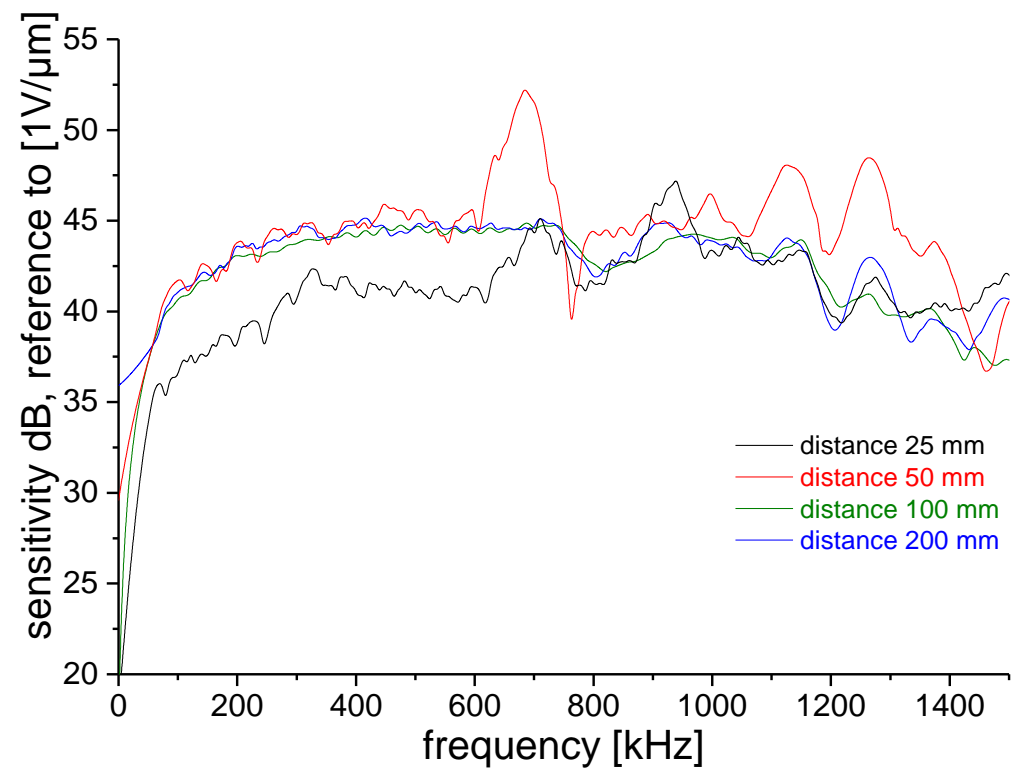

Fig. 15. Comparison of conical sensor sensitivity for steel plate with distances between source and sensor.

\subsubsection{Duration of signal evaluation}

While chosen constant in section 3.4.2, the next factor to consider is the duration for signal evaluation. Within an experimental setting, this potential aspect needs to be considered for evaluation of the Lamb wave signals. This is particularly relevant for Lamb waves, as other than the bulk wave and Rayleigh wave cases (cf. Figs. 5, 7), there is no intuitive termination within a reasonable duration, as low frequency parts of the wave tend to extend for a relatively long time, potentially interfering with edge reflections. In most technical 
applications, the low frequency portion of the wave is limited by the vertical bandwidth of the acquisition system (including the sensor) and the high-pass filters. Thus, the sufficiency of a particular duration with respect to the arrival of all relevant wavelengths needs to be evaluated.

We chose the setting of a $3 \mathrm{~mm}$ steel plate with signal detection at $100 \mathrm{~mm}$ distance as reference and selected five different durations to extract signal information (cf. Fig. 16). The shortest choice $(30 \mu \mathrm{s})$ covers the arrival of the $S_{0}$ mode and a part of the $A_{1}$ mode (shows with similar intensity from 700 to $1000 \mathrm{kHz}$, as the $S_{0}$ mode in Pseudo-Wigner-Ville distribution for 30 us), while the extended durations add more and more information due to the arrival of the $A_{0}$ mode. Except for the reduction in length, all signals are evaluated according to the description in section 3.1. Signals of z-displacement are identically reduced in duration as the examples seen in Fig. 16). As seen from the sensitivity spectra of Fig. 17, the $30 \mu$ s durationbased sensitivity is systematically different compared to the other curves. This is due to the use of the $S_{0}$ mode and $A_{1}$ mode information only, which is insufficient to excite the sensor in the lower frequency range below $200 \mathrm{kHz}$ and additionally adds some bias to the spectrum above $1200 \mathrm{kHz}$. Both of these effects decrease significantly after increasing the considered duration to $60 \mu \mathrm{s}$, incorporating an almost equal time portion of the $S_{0}$ mode and the $A_{0}$ mode (signal is now dominated by low frequency region of the $A_{0}$ mode). For the durations of $90 \mu \mathrm{s}$ and above, no significant difference between the spectra is observed. This behavior emphasizes the importance of proper signal time termination and indicates the use of an appropriate signal duration including portions of all Lamb wave modes in the relevant frequency range.

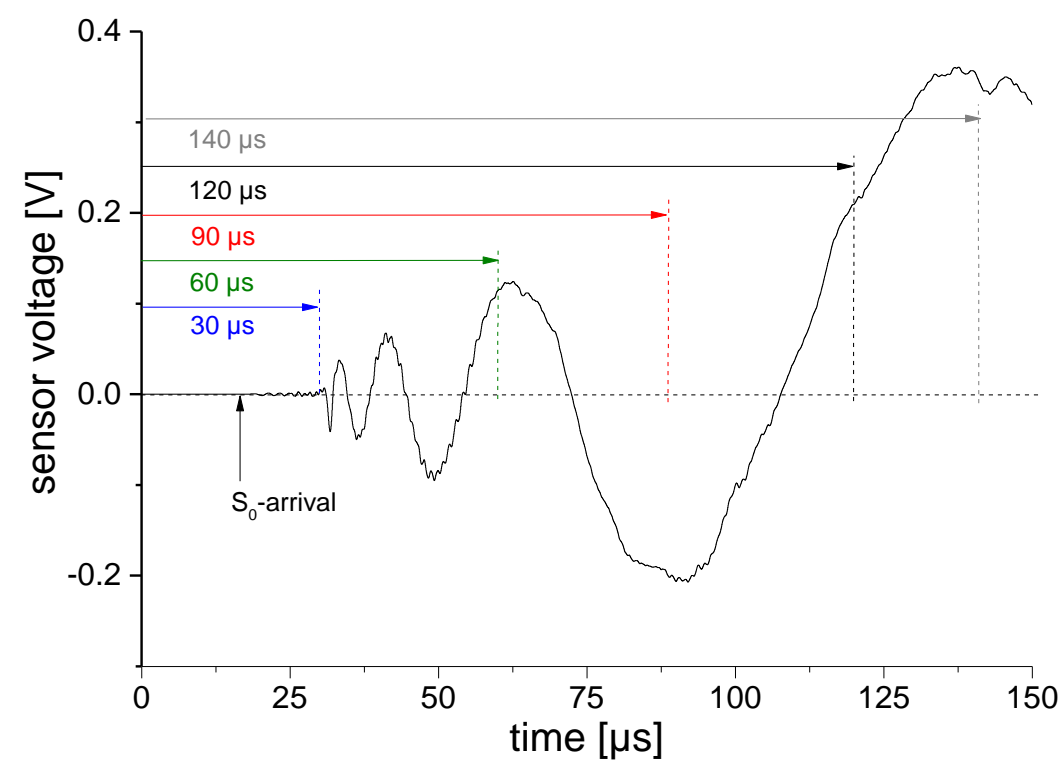

Fig. 16. Chosen time windows for signal evaluation for $3 \mathrm{~mm}$ steel plate at $100 \mathrm{~mm}$ propagation distance. 


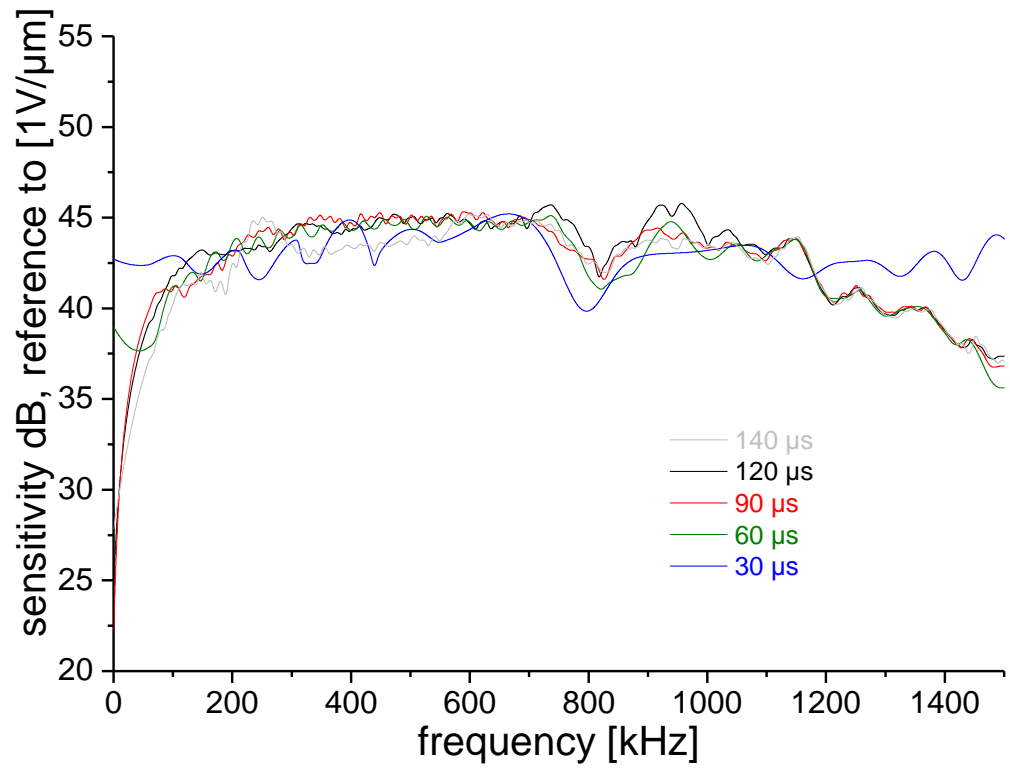

Fig. 17. Comparison of conical sensor sensitivity for $3 \mathrm{~mm}$ steel plate (sensor at $100 \mathrm{~mm}$ ) with different durations for signal evaluation.

\subsubsection{In-plane sources vs. out-of-plane sources}

In the two previous sections, the range of frequency content in the signals was found to affect the computed sensitivity spectrum. This investigation now turns to another possible factor that may influence sensitivity results. In absolute calibration approaches, the source direction is usually in the direction normal to the surface (out-of-plane source) and the sensor face. As a significant fraction of acoustic emission sources may cause an in-plane excitation, it is an interesting numerical experiment to analyze the sensor response for these two fundamentally different source types. Therefore, we modified the source direction of Fig. 1-d to act along the $x$-axis (i.e. towards the sensor and parallel to the sensor face). For a $100 \mathrm{~mm}$ propagation distance in a $3 \mathrm{~mm}$ steel plate, the sensor signals are shown in Fig. 18, and the corresponding sensitivity spectra are shown in Fig. 19. As expected from the change of source orientation [29], there is a significant change in the amplitude ratio of the $S_{0}$ mode and $A_{0}$ mode, being higher for the in-plane source (cf. Fig 18). With all signal evaluation settings identical and the same source strength, the sensitivity results have a fairly similar shape as a function of frequency, except for some $\pm 2 \mathrm{~dB}$ deviations at frequencies above $700 \mathrm{kHz}$. This behavior may partially be explained by the change in bandwidth caused by both source types. In particular, most noticeable deviations of the sensitivity curves occurs above $700 \mathrm{kHz}$, i.e. in the range of the dominant excitation of the $S_{0}$ mode, which is not as pronounced in the out-of-plane case. 
(a)
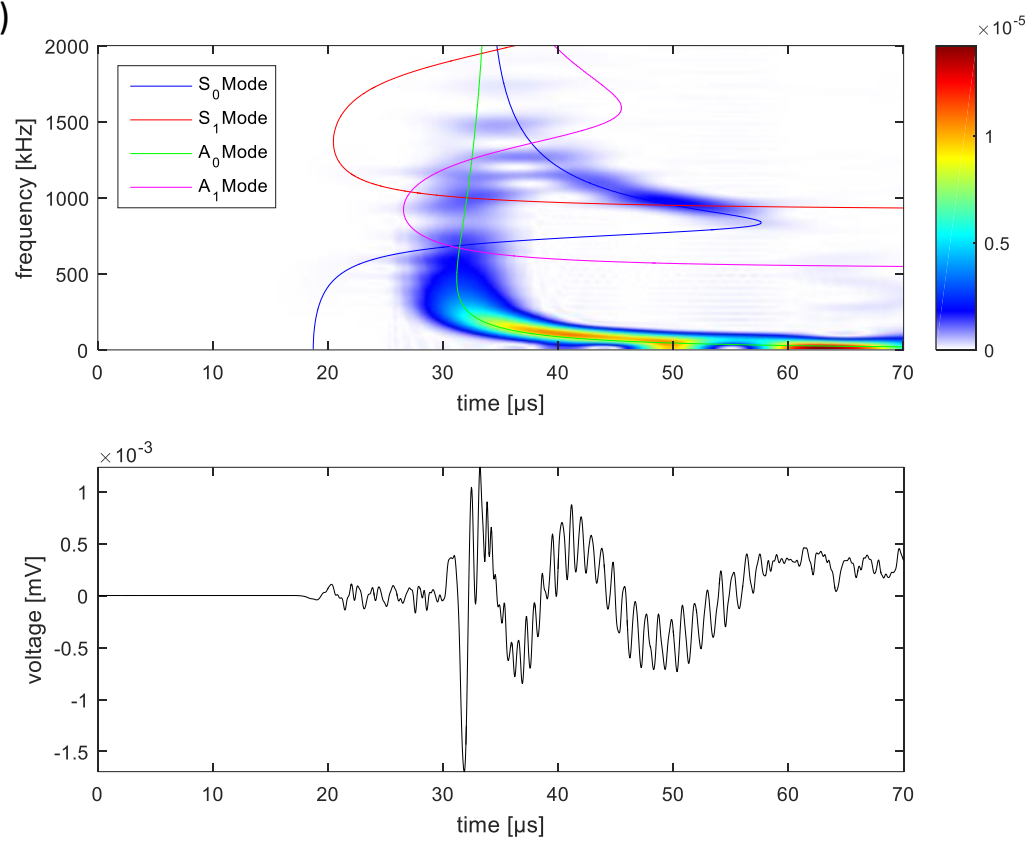

(b)
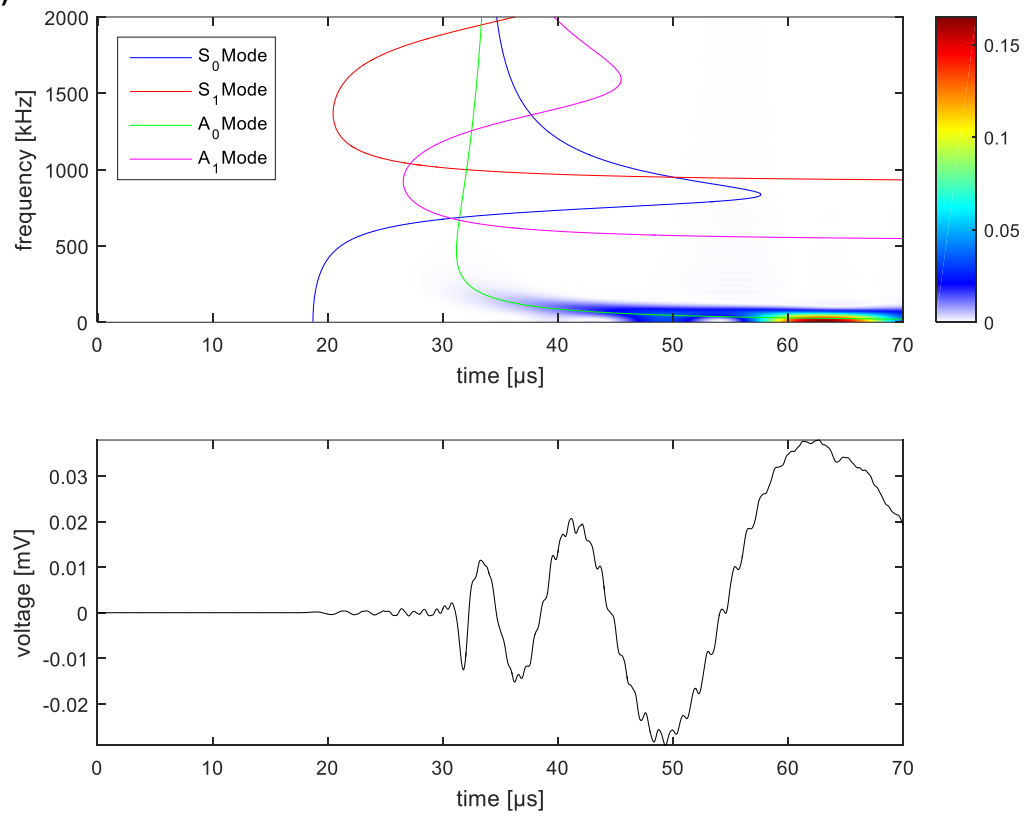

Fig. 18. Comparison of $A E$ sensor signals and smoothed Pseudo-Wigner-Ville distribution (with superimposed dispersions curves) for in-plane (a) and out-of-plane source (b). 


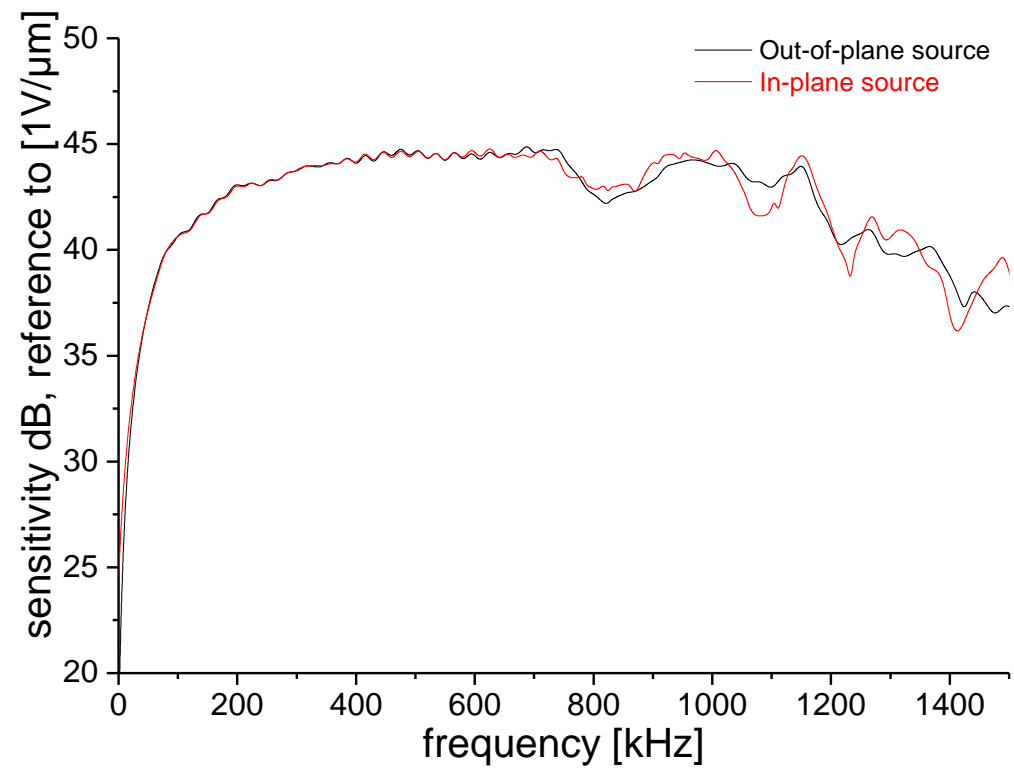

Fig. 19. Comparison of conical sensor sensitivity for steel plate excitation with in-plane and out-of-plane source.

\section{Concluding remarks}

In this study, we investigated the effect of different potential configurations for absolute acoustic emission sensor calibration. Based on the individual combination of acoustic impedances (propagation medium and sensor's contact surface material) there is no unique sensitivity curve of an acoustic emission sensor. Whilst for flat-with-frequency point-contact like sensor types (conical sensor) steel seems to provide very similar sensitivity curves for bulk, Rayleigh, rod and Lamb waves, this result does not seem to be applicable for aluminum or PMMA. For resonant sensor types with significant aperture (disc sensor), not even the steel medium did result in similar sensitivity curves for bulk, Rayleigh, rod and Lamb waves. For different propagation media, the change in response is credited to the change of electromechanical coupling impedances of the sensor elements in out-of-plane and in-plane direction. Thus, it seems reasonable to use a calibration procedure, which does not only consider the material aspect, but also considers the type of propagating wave. For technical applications involving Lamb waves, it seems more adequate to use plate geometries (properly implemented, as mentioned below) for such calibration procedures. This effectively allows overcoming the need for large bulk volumes as the calibration medium. For this plate case, the study results indicate technical feasibility of the calibration procedures, given a sufficient signal length is evaluated and interference of different wave modes at the detection point due to edge reflections is inhibited by using sufficiently large plates. However, careful selection of the plate thickness is required, as the change of Lamb wave modes has a significant influence on the measured detection sensitivity. 


\section{Literature}

[1] H. Hatano and E. Mori, "Acoustic-emission transducer and its absolute calibration," J. Acoust. Soc. Am., vol. 59, no. 2, pp. 344-349, Feb. 1976.

[2] F. R. Breckenridge, "Acoustic emission transducer calibration by means of the seismic surface pulse," J. Acoust. Emiss., vol. 1, no. 2, pp. 87-94, 1982.

[3] P. Theobald and R. Pocklington, "Velocity sensitivity calibration of AE sensors using the through wave method and laser interferometry," Ewgae 2010, 2010.

[4] C. B. Scruby and H. N. G. Wadley, "A calibrated capacitance transducer for the detection of acoustic emission," J. Phys. D. Appl. Phys., vol. 11, no. 11, pp. 1487-1494, Aug. 1978.

[5] G. C. McLaskey and S. D. Glaser, "Acoustic Emission Sensor Calibration for Absolute Source Measurements," J. Nondestruct. Eval., vol. 31, no. 2, pp. 157-168, Jan. 2012.

[6] S. V. Augutis, B. Muravin, and E. Vastakas, "Application of waveguides for the calibration of acoustic emission transducers," in 32nd European Conference on Acoustic Emission, 2016, pp. 7-14.

[7] B. Burks and M. A. Hamstad, "An Experimental-numerical Investigation of the Face-toface Sensor Characterization Technique," Mater. Eval., vol. 73, no. 3, 2015.

[8] M. Sause, Identification of failure mechanisms in hybrid materials utilizing pattern recognition techniques applied to acoustic emission signals. Berlin: mbv-Verlag, 2010.

[9] M. G. R. Sause, M. A. Hamstad, and S. Horn, "Finite element modeling of conical acoustic emission sensors and corresponding experiments," Sensors Actuators $A$ Phys., vol. 184, pp. 64-71, Sep. 2012.

[10] M. G. R. Sause, In Situ Monitoring of Fiber-Reinforced Composites, vol. 242. Cham: Springer International Publishing, 2016.

[11] L. Zhang and D. Ozevin, "Calibration of Acoustic Emission Sensors with Laser Ultrasound and Multi-Physics Modeling," in Progress in Acoustic Emission XVIII, 2016, pp. 269-274.

[12] L. Zhang, H. Yalcinkaya, and D. Ozevin, "Numerical approach to absolute calibration of piezoelectric acoustic emission sensors using multiphysics simulations," Sensors Actuators A Phys., vol. 256, pp. 12-23, Apr. 2017.

[13] F. R. Breckenridge and M. Greenspan, "Surface-Wave Displacement: Absolute Measurements using a Capacitance Transducer," J. Acoust. Soc. Am., vol. 69, no. 2, pp. 1177-1185, 1981.

[14] F. Breckenridge, T. Proctor, N. Hsu, S. Fick, and D. Eitzen, "Transient sources for acoustic emission work," in Progress in Acoustic Emission V, 1990, pp. 20-37.

[15] M. G. R. Sause, M. A. Hamstad, and S. Horn, "Finite element modeling of lamb wave propagation in anisotropic hybrid materials," Compos. Part B Eng., vol. 53, pp. 249257, 2013.

[16] B. Delaunay, "Sur la sphère vide. A la mémoire de Georges Voronoï," Bull. Acad. Sci. USSR 7, vol. 6, pp. 793-800, 1934.

[17] J. Chung and G. M. Hulbert, "A Time Integration Algorithm for Structural Dynamics With Improved Numerical Dissipation: The Generalized- $\alpha$ Method," J. Appl. Mech., vol. 60, no. 2, p. 371, 1993.

[18] B. F. Pouet and N. J. P. Rasolofosaon, "Measurement of broadband intrinsic ultrasonic attenuation and dispersion in solids with laser techniques," J. Acoust. Soc. Am., vol. 93, no. 3, pp. 1286-1292, Mar. 1993.

[19] M. A. Hamstad and C. M. Fortunko, "Development of practical wideband high-fidelity acoustic emission sensors," in Nondestructive Evaluation of Aging Infrastructure, 
1995, pp. 281-288.

[20] C. B. Scruby, "An introduction to acoustic emission," J. Phys. E., vol. 20, no. 8, p. 946, 1987.

[21] J. Waldmeyer, "Fast Fourier Transform for Step-Like Functions: The Synthesis of Three Apparently Different Methods," IEEE Trans. Instrum. Meas., vol. 29, no. 1, pp. 36-39, 1980.

[22] R. K. Miller and P. Mclntre, Eds., "Acoustic Emission Testing," in Nondestructive Testing Handbook, 2nd ed., V., Columbus, OH, 1987, pp. 126-132.

[23] L. Goujon and J. C. Baboux, "Behaviour of acoustic emission sensors using broadband calibration techniques," Meas. Sci. Technol., vol. 14, no. 7, pp. 903-908, Jul. 2003.

[24] G. C. McLaskey and S. D. Glaser, "Hertzian impact: experimental study of the force pulse and resulting stress waves.," J. Acoust. Soc. Am., vol. 128, no. 3, pp. 1087-96, Sep. 2010.

[25] Z. Heidary and D. Ozevin, "On the Influences of Boundary Reflections and Piezoelectric Sensors to the Characteristics of Elastic Waves for Pattern Recognition Methods," J. Nondestruct. Eval., vol. 34, no. 1, p. 271, Mar. 2015.

[26] M. A. Hamstad, A. O'Gallagher, and J. Gary, "Effects of Lateral Plate Dimensions on Acoustic Emission Signals from Dipole Sources," J. Acoust. Emiss., vol. 19, pp. 258-274, 2001.

[27] M. A. Hamstad, "Lamb Modal Regions With Significant AE Energy in a Thick Steel Plate," in International Conference on Acoutic Emission - Advances in Acoustic Emission, 2007, pp. 247-252.

[28] M. A. Hamstad, A. O'Gallagher, and J. Gary, "A wavelet transform applied to acoustic emission signals: Part 2: Source location," J. Acoust. Emiss., vol. 20, no. 853, pp. 6282, 2002.

[29] M. A. Hamstad, "Contrasts between the Acoustic Emission Signals Generated by Monopole Versus Dipole Sources," Adv. Mater. Res., vol. 13-14, pp. 61-68, 2006. 